\title{
Subdifferential Properties of Minimal Time Functions Associated with Set-Valued Mappings with Closed Convex Graphs in Hausdorff Topological Vector Spaces
}

\author{
Messaoud Bounkhel \\ Department of Mathematics, King Saud University, P.O. Box 2455, Riyadh 11451, Saudi Arabia \\ Correspondence should be addressed to Messaoud Bounkhel; bounkhel@ksu.edu.sa
}

Received 8 July 2013; Revised 30 August 2013; Accepted 2 September 2013

Academic Editor: Ismat Beg

Copyright (c) 2013 Messaoud Bounkhel. This is an open access article distributed under the Creative Commons Attribution License, which permits unrestricted use, distribution, and reproduction in any medium, provided the original work is properly cited.

For a set-valued mapping $M$ defined between two Hausdorff topological vector spaces $E$ and $F$ and with closed convex graph and for a given point $(x, y) \in E \times F$, we study the minimal time function associated with the images of $M$ and a bounded set $\Omega \subset F$ defined by $\mathscr{T}_{M, \Omega}(x, y):=\inf \{t \geq 0: M(x) \cap(y+t \Omega) \neq \emptyset\}$. We prove and extend various properties on directional derivatives and subdifferentials of $\mathscr{T}_{M, \Omega}$ at those points of $(x, y) \in E \times F$ (both cases: points in the graph $g$ ph $M$ and points outside the graph). These results are used to prove, in terms of the minimal time function, various new characterizations of the convex tangent cone and the convex normal cone to the graph of $M$ at points inside gph $M$ and to the graph of the enlargement set-valued mapping at points outside $g p h M$. Our results extend many existing results, from Banach spaces and normed vector spaces to Hausdorff topological vector spaces (Bounkhel, 2012; Bounkhel and Thibault, 2002; Burke et al., 1992; He and Ng, 2006; and Jiang and He 2009). An application of the minimal time function $\mathscr{T}_{M, \Omega}$ to the calmness property of perturbed optimization problems in Hausdorff topological vector spaces is given in the last section of the paper.

\section{Introduction}

Let $E$ and $F$ be two Hausdorff topological vector spaces and $\Omega$ a bounded nonempty subset of $F$. Let $M: E \rightrightarrows F$ be a setvalued mapping with nonempty closed values. We associate to with $M$ and $\Omega$ the so-called minimal time function defined by

$$
\begin{array}{r}
\mathscr{T}_{M, \Omega}(x, y):=\inf \{t \geq 0: M(x) \cap(y+t \Omega) \neq \emptyset\}, \\
\forall(x, y) \in E \times F .
\end{array}
$$

The case $M(x)=S$ is a fixed closed subset of $F$ that coincides with the minimal time function associated with $S$ and $\Omega$ and is defined by

$$
T_{S, \Omega}(u):=\inf \{t \geq 0: S \cap(u+t \Omega) \neq \emptyset\}, \quad \forall u \in F .
$$

This function $T_{S, \Omega}$ plays an important role in variational analysis, optimization, control theory, Hamilton-Jacobi partial differential equations, approximation theory, and so forth; the reader can find more discussions in [1-13] and the references therein. The value $\mathscr{T}_{M, \Omega}\left(x_{0}, y_{0}\right)$ can be seen as the minimal time $t \geq 0$ associated with the differential inclusion $\dot{y}(\tau) \in \Omega$, almost everywhere on $[0, t]$, with $y(0)=y_{0}$ and $y(t) \in M\left(x_{0}\right)$; in other words,

$$
\begin{aligned}
& \mathscr{T}_{M, \Omega}\left(x_{0}, y_{0}\right) \\
& =\left\{\begin{array}{cc}
\inf \{t>0: \exists y(\cdot) \text { s.t. } \dot{y}(\tau) \in \Omega, & \\
\text { a.e. }[0, t], y(0)=y_{0}, & \forall y_{0} \notin M\left(x_{0}\right), \\
\left.y(t) \in M\left(x_{0}\right)\right\}, & \forall y_{0} \in M\left(x_{0}\right) .
\end{array}\right.
\end{aligned}
$$

We notice that the minimal time function $\mathscr{T}_{M, \Omega}$ covers many crucial functions in variational analysis: the distance function to images $\Delta_{M}$, the indicator function, the usual distance function, and the Minkowski function, by taking some particular cases of $M$ and $\Omega$ as follows. 
(1) If $M(x)=\{0\}$, then $\mathscr{T}_{M, \Omega}(x, y)=T_{\{0\}, \Omega}(-y)=\rho_{\Omega}(y)$ which coincides with the Minkowski (gauge) function associated with $\Omega$; that is,

$$
\rho_{\Omega}(y)=\inf \{t \geq 0: y \in t \Omega\}, \quad \forall y \in F .
$$

(2) If $\Omega=\{0\}$, then $\mathscr{T}_{M, \Omega}(x, y)=T_{M(x),\{0\}}(y)=$ $\psi_{M(x)}(y)=\psi_{g p h M}(x, y)$, which coincides with the indicator function associated with $g p h M$. Here, $\psi_{S}(u)=0$ if $u \in S$ and $\psi_{S}(u)=+\infty$, otherwise.

(3) If $F$ is normed, $M(x)=\{0\}$, and $\Omega=\mathbb{B}_{F}$ is the closed unit ball in $F$, then $\mathscr{T}_{M, \Omega}(x, y)=T_{\{0\}, \mathbb{B}_{F}}(y)=\|y\|$.

(4) If $F$ is normed and $\Omega=\mathbb{B}_{F}$, then

$$
\mathscr{T}_{M, \Omega}(x, y)=\mathscr{T}_{M, \mathbb{B}}(x)=\inf _{s \in M(x)}\|y-s\|=\Delta_{M}(x, y)
$$

which coincides with the distance function associated with the images of $M$ (which has been successfully used in optimization theory, first by Clarke in [14] for Lipschitz set-valued mappings and later by various authors (see for instance the book [15] and the references therein) for set-valued mappings that are not necessarily locally Lipschitz).

(5) If $F$ is normed, $M(x)=S$, and $\Omega=\mathbb{B}_{F}$, then

$$
\mathscr{T}_{M, \Omega}(x, y)=T_{S, \mathbb{B}}(y)=\inf _{s \in S}\|y-s\|=d_{S}(y),
$$

which coincides with the usual distance function associated with $S$ in $F$.

From the above cases, we can see the importance of the study of the minimal time function $\mathscr{T}_{M, \Omega}$ in normed vector spaces as well as in Hausdorff topological vector spaces. This type of study will unify the study of all the above functions.

The case of minimal time function associated with a closed set $S$ has been the subject of many recent works [1$3,5-10,12,13]$. To the best of our knowledge, the unique work studying the function $\mathscr{T}_{M, \Omega}$ is [16], in which the author studied the Fréchet subdifferential of $\mathscr{T}_{M, \Omega}$ in Banach spaces. We mention that there are no results on the directional derivatives and subdifferentials of $\mathscr{T}_{M, \Omega}$ in the Hausdorff topological vector spaces. Starting from this point, as a goal, we will develop a thorough study of the minimal time function in Hausdorff topological vector spaces in the convex setting. The nonconvex case will be the subject of a series of forthcoming works by the author. In the present paper, we extend various existing results on directional derivatives and subdifferentials of $\mathscr{T}_{M, \Omega}$ and their relationships to tangent and normal cones in Hausdorff topological vector spaces. The paper is organized as follows. Section 2 is devoted to state the main notations and definitions used throughout the paper. In Section 3, we prove our main results for points on the graph of $M$. The case of points outside the graph of $M$ is studied in Section 4. In the last section we state an application of the minimal time function $\mathscr{T}_{M, \Omega}$ to the study of the calmness property of optimization problems in Hausdorff topological vector spaces.

\section{Notations and Preliminaries}

Throughout the whole paper (unless otherwise specified), we assume that $E$ and $F$ are two Hausdorff topological vector spaces. We will denote by $E^{*}$ and $F^{*}$ the topological dual of $E$ and $F$, respectively, and by $\langle(\cdot, \cdot),(\cdot, \cdot)\rangle$ the pairing between the spaces $E \times F$ and $E^{*} \times F^{*}$.

Let $S$ be a nonempty closed convex subset of $E \times F$ and $(\bar{x}, \bar{y})$ a point in $S$. The convex tangent cone $T(S ;(\bar{x}, \bar{y}))$ is defined by $T(S ;(\bar{x}, \bar{y}))=\operatorname{cl}\left[\mathbb{R}_{+}(S-(\bar{x}, \bar{y}))\right]$, where $\mathbb{R}_{+}$ denotes the set of all nonnegative real numbers. The convex normal cone to $S$ at $(\bar{x}, \bar{y})$ is defined as the negative polar of $T(S ;(\bar{x}, \bar{y}))$; that is, $N(S ;(\bar{x}, \bar{y})):=(T(S ;(\bar{x}, \bar{y})))^{-}$, where $A^{-}:=\left\{\left(x^{*}, y^{*}\right) \in E^{*} \times F^{*}:\left\langle\left(x^{*}, y^{*}\right),(h, k)\right\rangle \leq 0\right.$, for all $(h$, $k) \in A\}$, for any subset $A \subset E \times F$. Recall also from [14] that $N(S ;(\bar{x}, \bar{y}))$ is also characterized by $N(S ;(\bar{x}, \bar{y}))=\left\{\left(x^{*}, y^{*}\right) \epsilon\right.$ $E^{*} \times F^{*}:\left\langle\left(x^{*}, y^{*}\right),(x, y)-(\bar{x}, \bar{y})\right\rangle \leq 0$, for all $\left.(x, y) \in S\right\}$.

Let $f$ be a convex function from $E$ into $\mathbb{R} \cup\{+\infty\}$ with $\bar{u} \in \operatorname{dom} f:=\{u \in E: f(u)<\infty\}$; the directional derivative (resp., the convex subdifferential) of $f$ at $\bar{u}$ is defined by

$$
f^{\prime}(\bar{u} ; v):=\lim _{t \downarrow 0} t^{-1}[f(\bar{u}+t v)-f(\bar{u})], \quad \forall v \in E .
$$

(resp.,

$$
\begin{aligned}
& \partial f(\bar{x}) \\
& \quad:=\left\{u^{*} \in E^{*}:\left\langle u^{*}, v\right\rangle \leq f^{\prime}(\bar{u} ; v), \forall v \in E\right\} \\
& \left.\quad=\left\{u^{*} \in E^{*}:\left\langle u^{*}, u-\bar{u}\right\rangle \leq f(u)-f(\bar{u}), \forall u \in E\right\}\right) .
\end{aligned}
$$

Note that for 1.s.c convex functions we have $f^{\prime}(\bar{u} ; h)=$ $\sigma_{\partial f(\bar{u})}(h)$, whenever $\bar{u} \in \operatorname{core}(\operatorname{dom} f)$ and $E$ is a Hausdorff locally convex topological vector space. Here, $\sigma_{A}$ denotes the support function associated with a closed subset $A \subset E^{*}$ defined by $\sigma_{A}(h):=\sup \left\{\left\langle x^{*}, h\right\rangle: x^{*} \in A\right\}$ and core $(S):=$ $\left\{x \in E\right.$ : for all $h \in E, \exists t_{x, h}>0$, such that $\left.x+\left[0, t_{x, h}\right] h \subset S\right\}$ is called the algebraic interior of $S$ (for more details on the core, we refer the reader to [17]).

\section{Points on the Graph of the Set-Valued Mapping}

Before starting the study of minimal time functions for setvalued mappings with closed convex graphs, we need to prove some results for general set-valued mappings $M: E \rightrightarrows F$ with nonempty values (with graph not necessarily closed nor convex). These results have their own interests. We start with the following lemma which is needed in all the proofs of our work.

Lemma 1. Let $E$ and $F$ be Hausdorff topological vector spaces. Assume that $\Omega$ is a bounded set in $F$ and $M$ is a set-valued mapping with nonempty values in $F$.

(1) If $\mathscr{T}_{M, \Omega}(x, y)=0$, then $y \in \operatorname{cl}(M(x))$.

(2) Conversely, for any $y \in M(x)$, one has $T_{M, \Omega}(x, y)=0$.

(3) If in addition $0 \in \operatorname{int} \Omega$, then for any $y \in \operatorname{cl}(M(x))$ one has $T_{M, \Omega}(x, y)=0$. 
Proof. (1) Let $x \in E$ with $\mathscr{T}_{M, \Omega}(x, y)=0$, then for all $n \geq 1$, $\exists t_{n} \geq 0$ such that $\mathscr{T}_{M, \Omega}(x, y)+1 / n>t_{n} \geq \mathscr{T}_{M, \Omega}(x, y)$ with $M(x) \cap\left(y+t_{n} \Omega\right) \neq \emptyset$; that is, there exists $u_{n} \in \Omega$ such that $y+t_{n} u_{n} \in M(x)$. Let $V$ be any neighborhood of 0 in $F$. There exists $W$ as a balanced neighborhood of 0 such that $W \subset V$. Since $\Omega$ is bounded, there exists $\alpha>0$ such that $\Omega \subset \alpha W$. Hence, there exists $w_{n} \in W$ such that $u_{n}=\alpha w_{n}$. Clearly, for any $n>\alpha$, we have $t_{n} \alpha \in(0,1)$, and so by the fact that $W$ is balanced we obtain

$$
t_{n} u_{n}=t_{n} \alpha w_{n} \in t_{n} \alpha W \subset W \subset V .
$$

Since $V$ is arbitrary, we get the convergence of the sequence $t_{n} u_{n}$ to zero, that is, the convergence of the sequence $y+t_{n} u_{n}$ to $y$, and since $y+t_{n} u_{n} \in M(x)$, we get $y \in \operatorname{cl}(M(x))$.

(2) Let $y \in M(x)$ and let $w \in \Omega$. Clearly, we have $y=$ $y+0 \cdot w \in(y+0 \cdot \Omega) \cap M(x)$. This ensures by definition that $\mathscr{T}_{M, \Omega}(x, y)=0$.

(3) Assume now that $0 \in$ int $\Omega$. Let $y \in \operatorname{cl}(M(x))$ and fix any $\epsilon>0$. Clearly, $\epsilon \Omega \in \mathcal{N}(0)$, and hence, $(y+\epsilon \Omega) \cap$ $M(x) \neq \emptyset$. It follows that $0 \leq \mathscr{T}_{M, \Omega}(x, y) \leq \epsilon$ which ensures that $\mathscr{T}_{M, \Omega}(x, y)=0$.

Note that the assumption $0 \in \operatorname{int} \Omega$ in (3) cannot be removed when the values of $M$ are open sets. Take $M(x)$ as any open set, and take $\Omega=\{0\}$. Clearly, for all $y \in$ $\operatorname{bd}(M(x)):=\operatorname{cl}(M(x)) \backslash M(x)$, we have $\mathscr{T}_{M, \Omega}(x, y)=$ $\psi_{M(x)}(y)=+\infty$.

As a corollary of parts (1) and (2) in Lemma 1 we have the following.

Corollary 2. Let $E$ and $F$ be Hausdorff topological vector spaces, and let $M: E \rightrightarrows F$ be a set-valued mapping with nonempty closed values. Assume that $\Omega$ is a bounded set in $F$. Then $\mathscr{T}_{M, \Omega}(x, y)=0$ if and only if $y \in M(x)$.

The following lemma characterizes the convexity of the graph of set-valued mappings in terms of the convexity of its associated minimal time function in Hausdorff topological vector spaces. It extends the well known characterization of the convexity (see, for instance, the lemma on page 53 in [14]) in terms of the distance function in normed vector spaces as well as the one in terms of the indicator function.

Lemma 3. Let $E$ and $F$ be two Hausdorff topological vector spaces, $M: E \rightrightarrows F$ a set-valued mapping with nonempty closed values, and $\Omega$ a bounded convex set in $F$. Then, $\mathscr{T}_{M, \Omega}(\cdot, \cdot)$ is convex on its domain if and only if the graph gph $M$ is convex.

Proof. Assume that $\mathscr{T}_{M, \Omega}(\cdot, \cdot)$ is convex on its domain; that is,

$$
\begin{aligned}
\mathscr{T}_{M, \Omega} & \left(\lambda\left(x_{1}, y_{1}\right)+(1-\lambda)\left(x_{2}, y_{2}\right)\right) \\
\leq & \lambda \mathscr{T}_{M, \Omega}\left(x_{1}, y_{1}\right)+(1-\lambda) \mathscr{T}_{M, \Omega}\left(x_{2}, y_{2}\right),
\end{aligned}
$$

for all $\left(x_{1}, y_{1}\right),\left(x_{2}, y_{2}\right) \in \operatorname{dom} \mathscr{T}_{M, \Omega}$, and all $\lambda \in[0,1]$. Therefore, by Corollary 2 , for any $\left(x_{1}, y_{1}\right),\left(x_{2}, y_{2}\right) \in g p h M$, we have $\mathscr{T}_{M, \Omega}\left(x_{1}, y_{1}\right)=\mathscr{T}_{M, \Omega}\left(x_{2}, y_{2}\right)=0$, and so by the previous inequality, we obtain $\mathscr{T}_{M, \Omega}\left(\lambda\left(x_{1}, y_{1}\right)+(1-\right.$ $\left.\lambda)\left(x_{2}, y_{2}\right)\right)=0$, and hence by Corollary 2 , once again we get $\lambda y_{1}+(1-\lambda) y_{2} \in M\left(\lambda x_{1}+(1-\lambda) x_{2}\right)$; that is, $\lambda\left(x_{1}, y_{1}\right)+$ $(1-\lambda)\left(x_{2}, y_{2}\right) \in g p h M$, which means that $g p h M$ is convex. Conversely, let $\left(x_{i}, y_{i}\right) \in \operatorname{dom} \mathscr{T}_{M, \Omega}(i=1,2)$, and let any $\lambda \in[0,1]$. Fix any $\epsilon>0$. Since $\mathscr{T}_{M, \Omega}\left(x_{1}, y_{1}\right)<\infty$ and $\mathscr{T}_{M, \Omega}\left(x_{2}, y_{2}\right)<\infty$, we can find that $w_{i} \in \Omega,(i=1,2)$ and $t_{i} \geq 0(i=1,2)$ such that $t_{i}<\mathscr{T}_{M, \Omega}\left(x_{i}, y_{i}\right)+\epsilon$ and $y_{i}+t_{i} w_{i} \in M\left(x_{i}\right)(i=1,2)$. So the convexity of $g p h M$ ensures that $\lambda\left(x_{1}, y_{1}+t_{1} w_{1}\right)+(1-\lambda)\left(x_{2}, y_{2}+t_{2} w_{2}\right) \in g p h M$. Hence,

$$
\begin{gathered}
{\left[\lambda y_{1}+(1-\lambda) y_{2}\right]+\left[\lambda t_{1}+(1-\lambda) t_{2}\right] \bar{w}} \\
\in M\left(\lambda x_{1}+(1-\lambda) x_{2}\right),
\end{gathered}
$$

where $\bar{w}:=\left[\left(\lambda t_{1} /\left(\lambda t_{1}+(1-\lambda) t_{2}\right)\right) w_{1}+\left((1-\lambda) t_{2} /\left(\lambda t_{1}+(1-\right.\right.\right.$ $\left.\left.\lambda) t_{2}\right)\right) w_{2}$. On the other hand, by the convexity of $\Omega$, we have

$$
\bar{w}=\frac{\lambda t_{1}}{\lambda t_{1}+(1-\lambda) t_{2}} w_{1}+\frac{(1-\lambda) t_{2}}{\lambda t_{1}+(1-\lambda) t_{2}} w_{2} \in \Omega,
$$

which ensures that

$$
\begin{gathered}
\left(\left[\lambda y_{1}+(1-\lambda) y_{2}\right]+\left[\lambda t_{1}+(1-\lambda) t_{2}\right] \Omega\right) \\
\cap M\left(\lambda x_{1}+(1-\lambda) x_{2}\right) \neq \emptyset .
\end{gathered}
$$

This ensures that

$$
\begin{aligned}
\mathscr{T}_{M, \Omega} & \left(\lambda\left(x_{1}, y_{1}\right)+(1-\lambda)\left(x_{2}, y_{2}\right)\right) \\
& =\mathscr{T}_{M, \Omega}\left(\lambda x_{1}+(1-\lambda) x_{2}, \lambda y_{1}+(1-\lambda) y_{2}\right) \\
& \leq \lambda t_{1}+(1-\lambda) t_{2} \\
& <\lambda \mathscr{T}_{M, \Omega}\left(x_{1}, y_{1}\right)+(1-\lambda) \mathscr{T}_{M, \Omega}\left(x_{2}, y_{2}\right)+\epsilon,
\end{aligned}
$$

for any $\epsilon>0$. Thus, taking $\epsilon \rightarrow 0$ completes the proof.

Note that the convexity of $\Omega$, in the proof of Lemma 3, is needed only in one direction (reverse implication); that is, the convexity of $\mathscr{T}_{M, \Omega}$ ensures the convexity of the graph of $M$ even when $\Omega$ is not convex.

Now, we are looking for the lower semicontinuity of the minimal time function in Hausdorff topological vector spaces.

Proposition 4. Let $E$ and $F$ be two Hausdorff topological vector spaces. Assume that $\Omega$ is compact in $F$ and gph $M$ is closed in $E \times F$. Then $\mathscr{T}_{M, \Omega}$ is lower semicontinuous at any $(\bar{x}, \bar{y}) \in \operatorname{dom} \mathscr{T}_{M, \Omega}$.

Proof. Let $(\bar{x}, \bar{y}) \in \operatorname{dom} \mathscr{T}_{M, \Omega}$. We have to prove that

$$
\mathscr{T}_{M, \Omega}(\bar{x}, \bar{y}) \leq \liminf _{(x, y) \rightarrow(\bar{x}, \bar{y})} \mathscr{T}_{M, \Omega}(x, y) .
$$

The case $\lim \inf _{(x, y) \rightarrow(\bar{x}, \bar{y})} \mathscr{T}_{M, \Omega}(x, y)=\infty$ is obvious, so we assume that

$$
\alpha:=\liminf _{(x, y) \rightarrow(\bar{x}, \bar{y})} \mathscr{T}_{M, \Omega}(x, y)<\infty .
$$

Let $\left(x_{j}, y_{j}\right)_{j \in J}$ be a net satisfying the above lim inf; that is, $\left(x_{j}, y_{j}\right) \rightarrow(\bar{x}, \bar{y})$ and $\alpha=\lim _{j} \mathscr{T}_{M, \Omega}\left(x_{j}, y_{j}\right)$. Let $\left(t_{j}\right)_{j \in J}$ be a net of positive real numbers satisfying $t_{j} \rightarrow 0$. 
By the definition of the minimal time function, we can find for any $j \in J$ a real number $\delta_{j}$ such that

$$
\begin{gathered}
0 \leq \mathscr{T}_{M, \Omega}\left(x_{j}, y_{j}\right) \leq \delta_{j}<\mathscr{T}_{M, \Omega}\left(x_{j}, y_{j}\right)+t_{j}, \\
\left(y_{j}+\delta_{j} \Omega\right) \cap M\left(x_{j}\right) \neq \emptyset, \quad \forall j \in J .
\end{gathered}
$$

Hence, for any $j \in J$, there exists $w_{j} \in \Omega$ such that $\left(x_{j}, y_{j}+\right.$ $\left.\delta_{j} w_{j}\right) \in g p h M$. Using the compactness of $\Omega$, we get the convergence of a subnet of $\left(w_{j}\right)_{j}$ to some point $w \in \Omega$. Without loss of generality the subnet is still denoted by $\left(w_{j}\right)_{j}$. Therefore, $\left(x_{j}, y_{j}+\delta_{j} w_{j}\right) \rightarrow(\bar{x}, \bar{y}+\alpha w) \in g p h M$ (by the closedness of $g p h M)$. This shows that $(\bar{y}+\alpha \Omega) \cap M(\bar{x}) \neq \emptyset$, which ensures that $\mathscr{T}_{M, \Omega}(\bar{x}, \bar{y}) \leq \alpha$, and hence, the proof is complete.

An inspection of the proof of the previous proposition shows that the conclusion is still valid under the assumptions that $\Omega$ is weakly compact and $g p h M$ is weakly closed. Consequently, we have the two following corollaries.

Corollary 5. Let $E$ and $F$ be two Hausdorff topological vector spaces. Assume that $\Omega$ is weakly compact in $F$ and gph $M$ is weakly closed in $E \times F$. Then, $\mathscr{T}_{M, \Omega}$ is lower semicontinuous at any $(\bar{x}, \bar{y}) \in \operatorname{dom} \mathscr{T}_{M, \Omega}$.

Corollary 6. Assume that $F$ is a reflexive Banach space, $\Omega$ is closed convex bounded set in $F$, and gph $M$ is closed convex set in $E \times F$. Then $\mathscr{T}_{M, \Omega}$ is lower semicontinuous at any $(\bar{x}, \bar{y}) \epsilon$ $\operatorname{dom} \mathscr{T}_{S, \Omega}$.

The following lemma is technical and is needed in some forthcoming proofs. Its proof is straightforward.

Lemma 7. Let $E$ and $F$ be two Hausdorff topological vector spaces, $M: E \rightrightarrows F$ a set-valued mapping, and $\Omega$ a nonempty set in F. Then,

$$
\begin{aligned}
t^{-1} & \mathscr{T}_{M, \Omega}(x+t h, y+t k) \\
& =T_{t^{-1}[M(x+t h)-y], \Omega}(k), \quad \forall(x, y),(h, k) \in E \times F, \forall t>0 .
\end{aligned}
$$

Now, we are going to establish our main results of this section. We prove some formulas and relationships between the directional derivative and the convex subdifferential of $\mathscr{T}_{M, \Omega}$ and the convex tangent cone and the convex normal cone of $g p h M$ in Hausdorff topological vector spaces at points in $g p h M$. We associate with the set-valued mapping $M$ a new set-valued mapping $\widetilde{T}_{M}: E \rightrightarrows F$ with graph $g p h \widetilde{T}_{M}:=T(g p h M ;(\bar{x}, \bar{y}))$; that is, $k \in \widetilde{T}_{M}(h)$ if and only if $(h, k) \in T(g p h M ;(\bar{x}, \bar{y}))$.

Theorem 8. Let $E$ and $F$ be two Hausdorff topological vector spaces and $M: E \rightrightarrows F$ a set-valued mapping. Assume that gph $M$ is a nonempty closed convex set in $E \times F,(\bar{x}, \bar{y}) \in$ gph $M$, and $\Omega$ is a bounded convex set in $F$. Then, one has
(1)

$$
\begin{gathered}
\mathscr{T}_{M, \Omega}^{\prime}((\bar{x}, \bar{y}) ;(h, k)) \\
=\lim _{t \rightarrow 0^{+}} t^{-1} \mathscr{T}_{M, \Omega}(\bar{x}+t h, \bar{y}+t k) \\
=\lim _{t \rightarrow 0^{+}} T_{t^{-1}[M(\bar{x}+t h)-\bar{y}], \Omega}(k), \\
\forall(h, k) \in E \times F ;
\end{gathered}
$$

(2)

$$
\begin{aligned}
\partial \mathscr{T}_{M, \Omega} & (\bar{x}, \bar{y}) \\
= & N(\operatorname{gph} M ;(\bar{x}, \bar{y})) \\
& \cap\left\{\left(x^{*}, y^{*}\right) \in E^{*} \times F^{*}: \sigma_{\Omega}\left(-y^{*}\right) \leq 1\right\}
\end{aligned}
$$

(3) if, in addition, $(\bar{x}, \bar{y}) \in \operatorname{core}\left(\operatorname{dom} \mathscr{T}_{M, \Omega}\right), E \times F$ is locally convex, then

$$
\begin{array}{r}
\mathscr{T}_{M, \Omega}^{\prime}((\bar{x}, \bar{y}) ;(h, k))=\mathscr{T}_{\widetilde{T}_{M, \Omega}}(h, k), \\
\forall(h, k) \in E \times F ;
\end{array}
$$

$(4)$

$$
\begin{aligned}
& T(\operatorname{gph} M ;(\bar{x}, \bar{k})) \\
& =\left\{(h, k) \in E \times F: \mathscr{T}_{M, \Omega}^{\prime}((\bar{x}, \bar{y}) ;(h, k))=0\right\} ;
\end{aligned}
$$

(5) and

$$
\begin{aligned}
& N(\operatorname{gph} M ;(\bar{x}, \bar{y})) \\
& \quad=\mathrm{cl}_{w^{*}}\left(\mathbb{R}_{+} \partial \mathscr{T}_{M, \Omega}((\bar{x}, \bar{y}))\right) .
\end{aligned}
$$

Proof. (1) By Lemma 3, we have the convexity of $\mathscr{T}_{M, \Omega}$, and so $\mathscr{T}_{M, \Omega}^{\prime}((\bar{x}, \bar{y}) ;(h, k))$ is well defined and hence, Corollary 2 and Lemma 7 complete the proof of part (1).

(2) Let $\left(x^{*}, y^{*}\right) \in N(g p h M ;(\bar{x}, \bar{y}))$ with $\sigma_{\Omega}\left(-y^{*}\right) \leq$ 1. Then, by definition of the convex normal cone and by Corollary 2, we have

$$
\begin{array}{r}
\left\langle\left(x^{*}, y^{*}\right),(x-\bar{x}, y-\bar{y})\right\rangle \\
\leq 0=\mathscr{T}_{M, \Omega}(x, y)-\mathscr{T}_{M, \Omega}(\bar{x}, \bar{y}), \\
\forall(x, y) \in g p h M .
\end{array}
$$

Assume now that $(x, y) \notin g p h M$, and let $\epsilon>0$. If $\mathscr{T}_{M, \Omega}(x, y)=\infty$, then the previous inequality holds obviously. Assume that $\mathscr{T}_{M, \Omega}(x, y)<\infty$. By definition of minimal time function, there exists $\delta>0$ and $w \in \Omega$ such that

$$
\begin{aligned}
0 & <\mathscr{T}_{M, \Omega}(x, y) \\
& \leq \delta<\mathscr{T}_{M, \Omega}(x, y)+\epsilon \text { with } \quad y+\delta w \in M(x) .
\end{aligned}
$$



that

Hence, we get by the definition of convex normal cones

$$
\left\langle\left(x^{*}, y^{*}\right),(x, y+\delta w)-(\bar{x}, \bar{y})\right\rangle \leq 0 .
$$

Therefore, combining this inequality with the assumption $\sigma_{\Omega}\left(-y^{*}\right) \leq 1$, we obtain that

$$
\begin{aligned}
& \left\langle\left(x^{*}, y^{*}\right),(x-\bar{x}, y-\bar{y})\right\rangle \\
& \quad=\left\langle\left(x^{*}, y^{*}\right),(x-\bar{x}, y+\delta w-\bar{y})\right\rangle+\delta\left\langle-y^{*}, w\right\rangle \\
& \quad \leq \delta \sigma_{\Omega}\left(-y^{*}\right) \leq \delta<\mathscr{T}_{M, \Omega}(x, y)+\epsilon,
\end{aligned}
$$

which ensures, by taking $\epsilon \rightarrow 0$, that

$$
\begin{aligned}
& \left\langle\left(x^{*}, y^{*}\right),(x-\bar{x}, y-\bar{y})\right\rangle \\
& \quad \leq \mathscr{T}_{M, \Omega}(x, y)-\mathscr{T}_{M, \Omega}(\bar{x}, \bar{y}), \quad \forall(x, y) \notin g p h M .
\end{aligned}
$$

Thus, this inequality holds for any $(x, y) \in E \times F$ which means that $\left(x^{*}, y^{*}\right) \in \partial \mathscr{T}_{M, \Omega}(\bar{x}, \bar{y})$.

Conversely, let $\left(x^{*}, y^{*}\right) \in \partial \mathscr{T}_{M, \Omega}(\bar{x}, \bar{y})$. Then, by definition, we have

$$
\begin{aligned}
& \left\langle\left(x^{*}, y^{*}\right),(x, y)-(\bar{x}, \bar{y})\right\rangle \\
& \quad \leq \mathscr{T}_{M, \Omega}(x, y)-\mathscr{T}_{M, \Omega}(\bar{x}, \bar{y}), \quad \forall(x, y) \in E \times F .
\end{aligned}
$$

Using Corollary 2, we have

$$
\left\langle\left(x^{*}, y^{*}\right),(x, y)-(\bar{x}, \bar{y})\right\rangle \leq 0 \quad \forall(x, y) \in g p h M .
$$

This means that $\left(x^{*}, y^{*}\right) \in N(g p h M ;(\bar{x}, \bar{y}))$. We have to prove that $\sigma_{\Omega}\left(-y^{*}\right) \leq 1$. Let $w \in \Omega$ and $\delta>0$. Then, $\bar{y}=\bar{y}-\delta w+\delta w \in M(\bar{x}) \cap(\bar{y}-\delta w+\delta \Omega)$; that is, $M(\bar{x}) \cap$ $(\bar{y}-\delta w+\delta \Omega) \neq \emptyset$, which ensures that $\mathscr{T}_{M, \Omega}(\bar{x}, \bar{y}-\delta w) \leq \delta$. Therefore, by (28), we have

$$
\begin{aligned}
\left\langle-y^{*}, \delta w\right\rangle & =\left\langle\left(x^{*}, y^{*}\right),(\bar{x}, \bar{y}-\delta w)-(\bar{x}, \bar{y})\right\rangle \\
& \leq \mathscr{T}_{M, \Omega}(\bar{x}, \bar{y}-\delta w) \leq \delta,
\end{aligned}
$$

which ensures that

$$
\left\langle-y^{*}, w\right\rangle \leq 1, \quad \forall w \in \Omega .
$$

This gives $\sigma_{\Omega}\left(-y^{*}\right) \leq 1$, and hence, the proof of (2) is complete.

(3) Let $(h, k) \in E \times F$. Assume first that $(h, k) \in T(g p h M$; $(\bar{x}, \bar{y}))=g p h \widetilde{T}_{M}$. Then, by Corollary 2 , we have $\mathscr{T}_{\widetilde{T}_{M}, \Omega}(h$, $k)=0$. Also, we have $\left\langle\left(x^{*}, y^{*}\right),(h, k)\right\rangle \leq 0$, for all $\left(x^{*}, y^{*}\right) \in$ $N(\operatorname{gph} M,(\bar{x}, \bar{y}))$. Hence, by $(2)$, we have $\left\langle\left(x^{*}, y^{*}\right),(h, k)\right\rangle \leq$ 0 , for all $\left(x^{*}, y^{*}\right) \in \partial \mathscr{T}_{M, \Omega}(\bar{x}, \bar{y})$, and so $\sigma_{\partial \mathscr{T}_{M, \Omega}(\bar{x}, \bar{y})}(h, k) \leq$ 0 . Now we use the assumption $(\bar{x}, \bar{y}) \in \operatorname{core}\left(\operatorname{dom} \mathscr{T}_{M, \Omega}\right)$ to write $\mathscr{T}_{M, \Omega}^{\prime}((\bar{x}, \bar{y}) ;(h, k))=\sigma_{\partial \mathscr{T}_{M, \Omega}(\bar{x}, \bar{y})}(h, k)$ and so $\mathscr{T}_{M, \Omega}^{\prime}((\bar{x}, \bar{y}) ;(h, k)) \leq 0$. Hence by Part (1) we obtain the needed equality

$$
\mathscr{T}_{M, \Omega}^{\prime}((\bar{x}, \bar{y}) ;(h, k))=0=\mathscr{T}_{\widetilde{T}_{M}, \Omega}(h, k) .
$$

Assume now that $(h, k) \notin T(g p h M ;(\bar{x}, \bar{y}))$. Without loss of generality, we assume that $\mathscr{T}_{\widetilde{T}_{M}, \Omega}(h, k)<\infty$. Put $\alpha:=$ $\mathscr{T}_{\widetilde{T}_{M}, \Omega}(h, k)$. Fix any $\delta \geq 0$ with $(k+\delta \Omega) \cap \widetilde{T}_{M}(h) \neq \emptyset$. There exists $w \in \Omega$ such that $k+\delta w \in \widetilde{T}_{M}(h)$; that is, $(h, k+$ $\delta w) \in T(g p h M ;(\bar{x}, \bar{y}))$. Therefore, by Part (2), for any $\left(x^{*}, y^{*}\right) \in \partial \mathscr{T}_{M, \Omega}(\bar{x}, \bar{y})$, we have $\sigma_{\Omega}\left(-y^{*}\right) \leq 1$ and $\left(x^{*}, y^{*}\right) \in$ $N(\operatorname{gph} M ;(\bar{x}, \bar{y}))$, and hence, we get the following

$$
\begin{aligned}
& \left\langle\left(x^{*}, y^{*}\right),(h, k)\right\rangle \\
& \quad=\left\langle\left(x^{*}, y^{*}\right),(h, k+\delta w)\right\rangle+\delta\left\langle-y^{*}, w\right\rangle \\
& \leq \delta \sigma_{\Omega}\left(-y^{*}\right) \leq \delta, \quad \forall\left(x^{*}, y^{*}\right) \in \partial \mathscr{T}_{M, \Omega}(\bar{x}, \bar{y}) .
\end{aligned}
$$

This ensures that

$$
\mathscr{T}_{M, \Omega}^{\prime}((\bar{x}, \bar{y}),(h, k))=\sigma_{\partial \mathscr{T}_{M, \Omega}(\bar{x}, \bar{y})}(h, k) \leq \delta,
$$

and since $\delta$ is taken arbitrarily, we take the infimum to obtain that

$$
\begin{aligned}
\mathscr{T}^{\prime}{ }_{M, \Omega} & ((\bar{x}, \bar{y}),(h, k)) \\
\leq & \inf \left\{\delta>0: \text { such that }(k+\delta \Omega) \cap \widetilde{T}_{M}(h) \neq \emptyset\right\} \\
= & \mathscr{T}_{\widetilde{T}_{M}, \Omega}(h, k) .
\end{aligned}
$$

Let us prove the reverse inequality

$$
\mathscr{T}_{\widetilde{T}_{M}, \Omega}(h, k) \leq \mathscr{T}_{M, \Omega}^{\prime}((\bar{x}, \bar{y}),(h, k)) .
$$

Obviously, we assume that $\mathscr{T}_{M, \Omega}^{\prime}((\bar{x}, \bar{y}),(h, k))<\infty$. Put $\alpha:=\mathscr{T}_{M, \Omega}^{\prime}((\bar{x}, \bar{y}),(h, k))$. Let $\left(t_{j}\right)_{j}$ be a net of positive real numbers converging to zero and satisfying the limit in the definition of the directional derivative; that is, $\alpha=$ $\lim _{j} T_{t_{j}^{-1}\left[M\left(\bar{x}+t_{j} h\right)-\bar{y}\right], \Omega}(k)$. Let $\delta_{j}>0$ and $w_{j} \in \Omega$ such that

$$
\begin{gathered}
T_{t_{j}^{-1}\left[M\left(\bar{x}+t_{j} h\right)-\bar{y}\right], \Omega}(k) \leq \delta_{j}<T_{t_{j}^{-1}\left[M\left(\bar{x}+t_{j} h\right)-\bar{y}\right], \Omega}(k)+t_{j}, \\
k+\delta_{j} w_{j} \in t_{j}^{-1}\left[M\left(\bar{x}+t_{j} h\right)-\bar{y}\right]
\end{gathered}
$$

that is, $\left(h, k+\delta_{j} w_{j}\right) \in t_{j}^{-1}[g p h M-(\bar{x}, \bar{y})]$.

Since $g p h M$ is closed convex, we have $T(g p h M$; $(\bar{x}, \bar{y}))=\operatorname{cl}\left(\mathbb{R}_{+}[g p h M-(\bar{x}, \bar{y})]\right)$, and so $\left(h, k+\delta_{j} w_{j}\right) \epsilon$ $T(\operatorname{gph} M ;(\bar{x}, \bar{y}))$, for all $j$; that is, $k+\delta_{j} w_{j} \in \widetilde{T}_{M}(h)$, for all $j$.

On the other hand we clearly have $\widetilde{T}_{\widetilde{T}_{M}, \Omega}(h, k) \leq \rho_{\Omega}(s-$ $k$ ), for all $s \in \widetilde{T}_{M}(h)$. Consequently, we obtain for all $j$ that

$$
\begin{aligned}
\mathscr{T}_{\widetilde{T}_{M}, \Omega}(h, k) & \leq \rho_{\Omega}\left(\left(k+\delta_{j} w_{j}\right)-k\right)=\delta_{j} \rho_{\Omega}\left(w_{j}\right) \\
& \leq \delta_{j}<T_{t_{j}^{-1}\left[M\left(\bar{x}+t_{j} h\right)-\bar{y}\right], \Omega}(k)+t_{j}, \quad \forall j .
\end{aligned}
$$

Taking the limit on this inequality, we obtain that

$$
\mathscr{T}_{\widetilde{T}_{M}, \Omega}(h, k) \leq \mathscr{T}_{M, \Omega}^{\prime}((\bar{x}, \bar{y}) ;(h, k)),
$$

thus completing the proof of (3). 
(4) The inclusion $T(g p h M,(\bar{x}, \bar{y})) \subset\{(h, k) \in E \times F$ : $\left.\mathscr{T}_{M, \Omega}^{\prime}((\bar{x}, \bar{y}) ;(h, k))=0\right\}$ follows from the assertion (3) and Corollary 2. Conversely, let $(h, k) \in E \times F$ with $\mathscr{T}_{M, \Omega}^{\prime}((\bar{x}$, $\bar{y}) ;(h, k))=0$. Let $\left(t_{j}\right)$ be a net of positive real numbers converging to zero and satisfying the limit $\mathscr{T}_{M, \Omega}^{\prime}((\bar{x}, \bar{y}) ;(h, k))=$ $\lim _{t_{j} \rightarrow 0} \mathscr{T}_{t_{j}^{-1}\left[M\left(\bar{x}+t_{j} h\right)-\bar{y}\right], \Omega}(k)$. For any $j \in J$, we can find that $\delta_{j}>0$ and $w_{j} \in \Omega$ such that

$$
\begin{gathered}
\mathscr{T}_{t_{j}^{-1}\left[M\left(\bar{x}+t_{j} h\right)-\bar{y}\right], \Omega}(k) \leq \delta_{j}<\mathscr{T}_{t_{j}^{-1}\left[M\left(\bar{x}+t_{j} h\right)-\bar{y}\right], \Omega}(k)+t_{j}, \\
k+\delta_{j} w_{j} \in t_{j}^{-1}\left[M\left(\bar{x}+t_{j} h\right)-\bar{y}\right]
\end{gathered}
$$$$
\text { that is, }\left(h, k+\delta_{j} w_{j}\right) \in t_{j}^{-1}[g p h M-(\bar{x}, \bar{y})] \text {. }
$$

Since the graph of $M$ is closed convex, we obtain that

$$
\left(h, k+\delta_{j} w_{j}\right) \in t_{j}^{-1}[g p h M-(\bar{x}, \bar{y})] \subset T(g p h M ;(\bar{x}, \bar{y})) .
$$

On the other hand, we have $\lim _{j} \delta_{j}=$ $\lim _{t_{j} \rightarrow 0} \mathscr{T}_{t_{j}^{-1}\left[M\left(\bar{x}+t_{j} h\right)-\bar{y}\right], \Omega}(k)=0$, and hence, by the boundedness of the set $\Omega$, we get the convergence of $\delta_{j} w_{j}$ to zero. This ensures the convergence of $k+\delta_{j} w_{j} \rightarrow k$ which ensures by the closedness of the tangent cone that $(h, k) \in T(\operatorname{gph} M,(\bar{x}, \bar{y}))$, and hence, the proof of $(4)$ is finished.

(5) The inclusion $\mathrm{cl}_{w^{*}}\left(\mathbb{R}_{+} \partial \mathscr{T}_{M, \Omega}((\bar{x}, \bar{y}))\right) \subset N(g p h M$; $(\bar{x}, \bar{y}))$ follows directly from part (2). So we prove the reverse one. To do that, we start by proving the inclusion

$$
\left(\partial \mathscr{T}_{M, \Omega}((\bar{x}, \bar{y}))\right)^{-} \subset T(g p h M ;(\bar{x}, \bar{y})) .
$$

Fix any $(h, k) \in\left(\partial \mathscr{T}_{M, \Omega}((\bar{x}, \bar{y}))\right)^{-}$. Then

$$
\left\langle\left(x^{*}, y^{*}\right) ;(h, k)\right\rangle \leq 0, \quad \forall\left(x^{*}, y^{*}\right) \in \partial \mathscr{T}_{M, \Omega}((\bar{x}, \bar{y})) .
$$

Since $\partial \mathscr{T}_{M, \Omega}((\bar{x}, \bar{y})) \neq \emptyset$ (because $\left.0 \in \partial \mathscr{T}_{M, \Omega}((\bar{x}, \bar{y}))\right)$ and $(\bar{x}, \bar{y}) \in \operatorname{core}\left(\operatorname{dom} \mathscr{T}_{M, \Omega}\right)$, then $\mathscr{T}_{M, \Omega}^{\prime}((\bar{x}, \bar{y}) ;(h, k))=$ $\sigma_{\partial \mathscr{T}_{M, \Omega}((\bar{x}, \bar{y}))}(h, k)$. Thus

$$
\mathscr{T}_{M, \Omega}^{\prime}((\bar{x}, \bar{y}) ;(h, k))=\sigma_{\partial \mathscr{T}_{M, \Omega}((\bar{x}, \bar{y}))}(h, k) \leq 0 .
$$

Since the reverse inequality $\mathscr{T}_{M, \Omega}^{\prime}((\bar{x}, \bar{y}) ;(h, k)) \geq 0$ follows directly from part (1), then we get the equality form $\mathscr{T}_{M, \Omega}^{\prime}((\bar{x}, \bar{y}) ;(h, k))=0$, and hence, part (4) ensures that $(h, k) \in T(\operatorname{gph} M ;(\bar{x}, \bar{y}))$. Therefore, $\left(\partial \mathscr{T}_{M, \Omega}((\bar{x}, \bar{y}))\right)^{-} \subset$ $T(\operatorname{gph} M ;(\bar{x}, \bar{y}))$. Since clearly we have $\left(\partial \mathscr{T}_{M, \Omega}((\bar{x}, \bar{y}))\right)^{-}=$ $\left(\mathrm{cl}_{w^{*}}\left(\mathbb{R}_{+} \partial \mathscr{T}_{M, \Omega}((\bar{x}, \bar{y}))\right)\right)^{-}$, then

$$
\left(\operatorname{cl}_{w^{*}}\left(\mathbb{R}_{+} \partial \mathscr{T}_{M, \Omega}((\bar{x}, \bar{y}))\right)\right)^{-} \subset T(g p h M ;(\bar{x}, \bar{y})),
$$

which ensures that

$$
\begin{aligned}
N(\operatorname{gph} M ;(\bar{x}, \bar{y})) & (T(\operatorname{gph} M ;(\bar{x}, \bar{y})))^{-} \\
& \subset\left(\left(\operatorname{cl}_{w^{*}}\left(\mathbb{R}_{+} \partial \mathscr{T}_{M, \Omega}((\bar{x}, \bar{y}))\right)\right)^{-}\right)^{-} \\
= & \mathrm{cl}_{w^{*}}\left(\mathbb{R}_{+} \partial \mathscr{T}_{M, \Omega}((\bar{x}, \bar{y}))\right) .
\end{aligned}
$$

The last equality follows from the fact that $E \times F$ is locally convex. Thus, the proof of the theorem is complete.

Many corollaries can be deduced from this theorem. We state the following one [18] by taking $\Omega$ to be the closed unit ball of a normed vector space $F$ and $M$ to be a constant setvalued mapping; that is, $M(x)=S$.

Corollary 9. Assume that $F$ is a normed vector space, $S$ is a closed convex subset in $F$, and $\bar{y} \in S$. Then, one has

$$
\begin{aligned}
d_{S}^{\prime}(\bar{y} ; k) & =\lim _{t \rightarrow 0^{+}} t^{-1} d_{S}(\bar{y}+t k) \\
& =\lim _{t \rightarrow 0^{+}} d_{t^{-1}[S-\bar{y}]}(k) \quad \forall k \in F ; \\
\text { (2) } \quad \partial d_{S}(\bar{y}) & =N(S ; \bar{y}) \cap\left\{y^{*} \in F^{*}:\left\|y^{*}\right\| \leq 1\right\} ; \\
\text { (3) } \quad d_{S}^{\prime}(\bar{y} ; k) & =d_{T(S ; \bar{y})}(k), \quad \forall k \in F ; \\
\text { (4) } T(S ; \bar{y}) & =\left\{k \in F: d_{S}^{\prime}(\bar{y} ; k)=0\right\} .
\end{aligned}
$$

\section{Points outside the Graph of the Set-Valued Mapping}

Now, we consider those points outside the graph of set-valued mappings $M$ with nonempty closed values. Let $(\bar{x}, \bar{y}) \notin$ gph M. Clearly, by Corollary 2, we have $\mathscr{T}_{M, \Omega}(\bar{x}, \bar{y})>0$. Denote $r:=\mathscr{T}_{M, \Omega}(\bar{x}, \bar{y})>0$, and define the enlargement set-valued mapping

$$
M_{r}(x):=\left\{y \in F: \mathscr{T}_{M, \Omega}(x, y) \leq r\right\} .
$$

Clearly, the graph of $M_{r}$ is defined as $g p h M_{r}:=\{(x, y) \epsilon$ $\left.E \times F: \mathscr{T}_{M, \Omega}(x, y) \leq r\right\}$. The following lemma is needed. It extends many existing results from normed spaces to Hausdorff topological vector spaces and from the case of sets to the case of set-valued mapping (see for instance $[15,19]$ ). Obviously, we have $\bar{y} \in M_{r}(\bar{x})$ and $M(\bar{x}) \subset M_{r}(\bar{x})$. We have also to point out that, due to Lemma 3, the convexity of the graph $g$ ph $M$ ensures the convexity of the graph of $M_{r}$ whenever $\Omega$ is bounded convex. However, the l.s.c. of $\mathscr{T}_{M, \Omega}$ ensures the closedness of the graph of $g p h M_{r}$. It follows then, from Lemma 3, the convexity of the minimal time function $\mathscr{T}_{M_{r}, \Omega}$ whenever $\mathscr{T}_{M, \Omega}$ is lower semicontinuous and $\Omega$ is bounded convex.

Lemma 10. Let $E$ and $F$ be two Hausdorff topological vector spaces. Assume that $M$ has nonempty closed values, $(\bar{x}, \bar{y}) \notin$ gph $M$, and $\Omega$ is a bounded convex set. Then, for any $(x, y) \in$ $E \times F$ we have

$$
\mathscr{T}_{M, \Omega}(x, y) \leq \mathscr{T}_{M_{r}, \Omega}(x, y)+r
$$

and for any $(x, y) \notin g p h M_{r}$ we have

$$
\mathscr{T}_{M, \Omega}(x, y)=\mathscr{T}_{M_{r}, \Omega}(x, y)+r .
$$

Proof. First we prove the inequality

$$
\mathscr{T}_{M, \Omega}(\bar{x}, y) \leq \mathscr{T}_{M_{r}, \Omega}(\bar{x}, y)+r, \quad \text { for any } y \in F .
$$


Assume that $(x, y) \in E \times F$ with $\mathscr{T}_{M_{r}, \Omega}(x, y)<\infty$. The case $\mathscr{T}_{M_{r}, \Omega}(x, y)=\infty$ is obvious. Fix any $\delta \geq 0$ satisfying $[y+$ $\delta \Omega] \cap M_{r}(x) \neq \emptyset$. Then, there exists $w \in \Omega$ such that $y+\delta w \in$ $M_{r}(x)$; that is, $\mathscr{T}_{M, \Omega}(x, y+\delta w) \leq r$. Then, for any $\epsilon>0$, we can choose $\lambda>0$ and $z \in \Omega$ such that

$$
\begin{aligned}
& \mathscr{T}_{M, \Omega}(x, y+\delta w) \\
& \leq \lambda<\mathscr{T}_{M, \Omega}(x, y+\delta w)+\epsilon, \quad y+\delta w+\lambda z \in M(x) .
\end{aligned}
$$

By convexity of $\Omega$, we have $\delta w+\lambda z \in(\delta+\lambda) \Omega$, and hence,

$$
y+\delta w+\lambda z \in M(x) \cap[y+(\delta+\lambda) \Omega] \neq \emptyset .
$$

Therefore,

$$
\begin{aligned}
\mathscr{T}_{M, \Omega}(x, y) & \leq \delta+\lambda \\
& <\delta+\mathscr{T}_{M, \Omega}(x, y+\delta w)+\epsilon \\
& <\delta+r+\epsilon .
\end{aligned}
$$

Since $\delta$ is taken arbitrarily satisfying $(y+\delta \Omega) \cap M_{r}(x) \neq \emptyset$, we obtain by taking the infimum over all $\delta$ the inequality

$$
\mathscr{T}_{M, \Omega}(x, y) \leq \mathscr{T}_{M_{r}, \Omega}(x, y)+r+\epsilon, \quad \forall \epsilon>0,
$$

and by taking $\epsilon \rightarrow 0$ we get the first desired inequality

$$
\mathscr{T}_{M, \Omega}(x, y) \leq \mathscr{T}_{M_{r}, \Omega}(x, y)+r .
$$

Assume now that $y \notin M_{r}(x)$, and let us prove the reverse inequality; that is, $\mathscr{T}_{M_{r}, \Omega}(x, y)+r \leq \mathscr{T}_{M, \Omega}(x, y)$. The case $\mathscr{T}_{M, \Omega}(x, y)=\infty$ is obvious so we suppose that $\mathscr{T}_{M, \Omega}(x, y)<$ $\infty$. Take arbitrarily a nonnegative number $\delta \geq 0$ for which $[y+\delta \Omega] \cap M(x) \neq \emptyset$. Then, there exists $w \in \Omega$ such that $y+$ $\delta w \in M(x)$. Clearly, $\mathscr{T}_{M, \Omega}(x, y) \leq \delta$, and since $y \notin M_{r}(x)$, we have $\delta>r$. Therefore,

$$
y+(\delta-r) w+r w \in M(x) \cap[y+(\delta-r) w+r \Omega] \neq \emptyset
$$

and so

$$
\begin{aligned}
& \mathscr{T}_{M, \Omega}(x, y+(\delta-r) w) \\
& \quad \leq r, \quad \text { that is, } y+(\delta-r) w \in M_{r}(x) .
\end{aligned}
$$

Thus,

$$
y+(\delta-r) w \in M_{r}(x) \cap[y+(\delta-r) \Omega] \neq \emptyset .
$$

Then,

$$
\mathscr{T}_{M_{r}, \Omega}(x, y) \leq \delta-r
$$

Since $\delta$ is taken arbitrarily with $[y+\delta \Omega] \cap M(x) \neq \emptyset$, we can take the infimum over all those $\delta$, and hence, we obtain that

$$
\mathscr{T}_{M_{r}, \Omega}(x, y)+r \leq \mathscr{T}_{M, \Omega}(x, y) .
$$

The proof is complete.
The previous lemma extends Lemma 3.4 in [2] from the case of sets to the case of set-valued mappings in Hausdorff topological vector spaces. Also, it extends the inequality (4.41) on page 97 in [15] from the case where $F$ is a normed vector space to the case where $F$ is a Hausdorff topological vector space and from the case of distance function to images $\Delta_{M}$ to the case of minimal time function $\mathscr{T}_{M, \Omega}$.

The first consequence of Lemma 10 is the following proposition in which we establish a relationship between the directional derivatives of $\mathscr{T}_{M, \Omega}$ and $\mathscr{T}_{M_{r}, \Omega}$.

Proposition 11. Let $E$ and $F$ be two Hausdorff topological vector spaces. Assume that $M$ has closed convex graph, $(\bar{x}, \bar{y}) \notin$ gph $M$, and $\Omega$ is a bounded convex set. Let $r:=\mathscr{T}_{M, \Omega}(\bar{x}, \bar{y})>$ 0 . Then, for any $(h, k) \in E \times F$, one has

$$
\mathscr{T}_{M, \Omega}^{\prime}((\bar{x}, \bar{y}) ;(h, k)) \leq \mathscr{T}_{M_{r}, \Omega}^{\prime}((\bar{x}, \bar{y}) ;(h, k)) .
$$

Proof. It follows, directly from the first part of the previous lemma, the fact that $\mathscr{T}_{M_{r}, \Omega}(\bar{x}, \bar{y})=0$ and the definition of the directional derivative.

In the following theorem, we characterize the convex tangent cone of the graph of the enlargement set-valued mapping $M_{r}$ as the set of all directions $(h, k)$ in $E \times F$ for which the directional derivative of $T_{M, \Omega}$ is nonnegative.

Theorem 12. Let $E$ and $F$ be two Hausdorff topological vector spaces. Assume that $M$ has a closed convex graph, $(\bar{x}, \bar{y}) \notin$ gph $M$, and $\Omega$ is a bounded convex set. Assume that $\mathscr{T}_{M, \Omega}$ is l.s.c. at $(\bar{x}, \bar{y})$. Then, one has

$$
\begin{aligned}
T & \left(g p h M_{r} ;(\bar{x}, \bar{y})\right) \\
& =\left\{(h, k) \in E \times F: \mathscr{T}_{M_{r}, \Omega}^{\prime}((\bar{x}, \bar{y}) ;(h, k))=0\right\} \\
& =\left\{(h, k) \in E \times F: \mathscr{T}_{M, \Omega}^{\prime}((\bar{x}, \bar{y}) ;(h, k)) \leq 0\right\} .
\end{aligned}
$$

Proof. The first equality follows directly from part (4) in Theorem 8. The direct inclusion of the second equality follows directly from the previous proposition. So it remains to prove the inclusion

$$
\begin{aligned}
& \left\{(h, k) \in E \times F: \mathcal{T}_{M, \Omega}^{\prime}((\bar{x}, \bar{y}) ;(h, k)) \leq 0\right\} \\
& \quad \subset T\left(g p h M_{r} ;(\bar{x}, \bar{y})\right) .
\end{aligned}
$$

Let $(h, k) \in E \times F$ with $\mathscr{T}_{M, \Omega}^{\prime}((\bar{x}, \bar{y}) ;(h, k)) \leq 0$. Let $\left(t_{j}\right)_{j}$ be a net in $(0, \infty)$ converging to 0 and satisfying the limit

$$
\begin{aligned}
& \mathscr{T}_{M, \Omega}^{\prime}((\bar{x}, \bar{y}) ;(h, k)) \\
& \quad=\lim _{j} t_{j}^{-1}\left[\mathscr{T}_{M, \Omega}\left(\bar{x}+t_{j} h, \bar{y}+t_{j} k\right)-\mathscr{T}_{M, \Omega}(\bar{x}, \bar{y})\right] .
\end{aligned}
$$

Use the lower semicontinuity of $\mathscr{T}_{M, \Omega}$ at $(\bar{x}, \bar{y})$ and the assumption $\bar{y} \notin M(\bar{x})$ to find some $j_{0} \in J$ such that

$$
\mathscr{T}_{M, \Omega}\left(\bar{x}+t_{j} h, \bar{y}+t_{j} k\right)>2 t_{j}^{2}>0, \quad \text { for any } j \geq j_{0} .
$$


Using now the assumption $\mathscr{T}_{M, \Omega}^{\prime}((\bar{x}, \bar{y}) ;(h, k)) \leq 0$ ensures the existence of some $j_{1} \geq j_{0}$ such that

$\mathscr{T}_{M, \Omega}\left(\bar{x}+t_{j} h, \bar{y}+t_{j} k\right) \leq r+t_{j}^{2}, \quad$ for any $j \geq j_{1}$.

Thus, combining (66) and (67), we obtain

$0<2 t_{j}^{2}<\mathscr{T}_{M, \Omega}\left(\bar{x}+t_{j} h, \bar{y}+t_{j} k\right) \leq r+t_{j}^{2}, \quad$ for any $j \geq j_{1}$.

Use now the definition of the minimal time function to choose for any $j \geq j_{1}$ some $w_{j} \in \Omega$ and $\delta_{j} \geq 0$ such that

$$
\begin{gathered}
\mathscr{T}_{M, \Omega}\left(\bar{x}+t_{j} h, \bar{y}+t_{j} k\right) \leq \delta_{j}<\mathscr{T}_{M, \Omega}\left(\bar{x}+t_{j} h, \bar{y}+t_{j} k\right)+t_{j}^{2}, \\
\bar{y}+t_{j} k+\delta_{j} w_{j} \in M\left(\bar{x}+t_{j} h\right) .
\end{gathered}
$$

Hence,

$$
0 \leq \delta_{j}-2 t_{j}^{2}<r, \quad \forall j \geq j_{1}
$$

Let $z_{j}:=\bar{y}+t_{j} k+2 t_{j}^{2} w_{j}$ and $k_{j}:=k+2 t_{j} w_{j}$. Then,

$$
\begin{gathered}
z_{j}+\left(\delta_{j}-2 t_{j}^{2}\right) w_{j}=\bar{y}+t_{j} k+\delta_{j} w_{j} \in M\left(\bar{x}+t_{j} h\right) \\
\cap\left(z_{j}+\left(\delta_{j}-2 t_{j}^{2}\right) \Omega\right) \neq \emptyset .
\end{gathered}
$$
$\left.t_{j} h\right)$.

So $\mathscr{T}_{M, \Omega}\left(\bar{x}+t_{j} h, z_{j}\right) \leq \delta_{j}-2 t_{j}^{2} \leq r$; that is, $z_{j} \in M_{r}(\bar{x}+$

Therefore,

$$
\bar{y}+t_{j} k_{j}=\bar{y}+t_{j} k+2 t_{j}^{2} w_{j} \in M_{r}\left(\bar{x}+t_{j} h\right),
$$

and so

$$
\left(h, k_{j}\right) \in t_{j}^{-1}\left[g p h M_{r}-(\bar{x}, \bar{y})\right] \subset T\left(g p h M_{r} ;(\bar{x}, \bar{y})\right) .
$$

Now, the boundedness of $\Omega$ ensures the convergence of $2 t_{j} w_{j}$ to zero, which gives the convergence of $k_{j}$ to $k$. Thus, by the closedness of the convex tangent cone, we get $(h, k) \epsilon$ $T\left(\operatorname{gph} M_{r},(\bar{x}, \bar{y})\right)$, and hence, the proof is finished.

Various corollaries can be deduced from the above theorem by taking special cases of $M$ and $\Omega$ and the spaces $E$ and $F$. We state the following one which has been proved in Corollary 4.4 in [15] (see also [20]) by taking $F$ to be a normed vector space, $\Omega$ to be the closed unit ball of $F$, and $M$ to be a constant set-valued mapping; that is, $M(x)=S$. In this case, $M_{r}(x)$ coincides with the enlargement set $S(r)$ defined by $S(r)=\left\{y \in F: d_{S}(y) \leq r\right\}$.

Corollary 13. Let $F$ be a normed vector space, $S$ a closed convex subset in $F$, and $\bar{y} \in S$. Then, one has

$$
\begin{aligned}
d_{S}^{\prime}(\bar{y} ; \bar{k}) \leq d_{S(r)}^{\prime}(\bar{y} ; \bar{k}) \leq d_{T(S(r) ; \bar{y})}(\bar{k}), \quad \forall \bar{k} \in F \\
T(S(r) ; \bar{y})=\left\{k \in F: d_{S(r)}^{\prime}(\bar{y} ; \bar{k})=0\right\} \\
=\left\{k \in F: d_{S}^{\prime}(\bar{y} ; \bar{k}) \leq 0\right\}
\end{aligned}
$$

Now, we turn to establish some relationships between the convex normal cone to $g p h M_{r}$ and the convex subdifferential of $\mathscr{T}_{M, \Omega}$ at $(\bar{x}, \bar{y}) \notin g p h M$.

Theorem 14. Let $E$ and $F$ Hausdorff topological vector spaces, $M$ a set-valued mapping with nonempty closed values, $\Omega$ a bounded convex set in $E$, and $(\bar{x}, \bar{y}) \notin g$ gh $M$. Assume that $\mathscr{T}_{M, \Omega}$ is l.s.c. at $(\bar{x}, \bar{y}), E$ and $F$ are locally convex, and $\partial \mathscr{T}_{M, \Omega}((\bar{x}, \bar{y})) \neq \emptyset$. Then,

$$
\begin{aligned}
N\left(\operatorname{gph} M_{r} ;(\bar{x}, \bar{y})\right) & =\mathrm{cl}_{w^{*}}\left(\mathbb{R}_{+} \partial \mathscr{T}_{M_{r}, \Omega}((\bar{x}, \bar{y}))\right) \\
& =\mathrm{cl}_{w^{*}}\left(\mathbb{R}_{+} \partial \mathscr{T}_{M, \Omega}((\bar{x}, \bar{y}))\right) .
\end{aligned}
$$

Proof. The first equality follows directly from Theorem 8. We will prove the equality $N\left(g p h M_{r} ;(\bar{x}, \bar{y})\right)=$ $\mathrm{cl}_{w^{*}}\left(\mathbb{R}_{+} \partial \mathscr{T}_{M, \Omega}((\bar{x}, \bar{y}))\right)$. Assume that $\partial \mathscr{T}_{M, \Omega}(\bar{x}, \bar{y}) \neq \emptyset$. First, we prove that

$$
\partial \mathscr{T}_{M, \Omega}(\bar{x}, \bar{y}) \subset N\left(\operatorname{gph} M_{r} ;(\bar{x}, \bar{y})\right) .
$$

Since $\partial^{C} \mathscr{T}_{M, \Omega}(\bar{x}, \bar{y}) \neq \emptyset$, we take some $\left(x^{*}, y^{*}\right) \in E^{*} \times F^{*}$ such that

$$
\begin{aligned}
& \left\langle\left(x^{*}, y^{*}\right),(h, k)\right\rangle \\
& \quad \leq \mathscr{T}_{M, \Omega}^{\prime}((\bar{x}, \bar{y}) ;(h, k)), \quad \forall(h, k) \in E \times F .
\end{aligned}
$$

Hence, for any $(h, k) \in T\left(g p h M_{r} ;(\bar{x}, \bar{y})\right)$, we have $\mathscr{T}_{M, \Omega}^{\prime}((\bar{x}, \bar{y}) ;(h, k)) \leq 0$ (by Theorem 12 ), and so

$$
\left\langle\left(x^{*}, y^{*}\right),(h, k)\right\rangle \leq \mathscr{T}_{M, \Omega}^{\prime}((\bar{x}, \bar{y}) ;(h, k)) \leq 0 ;
$$

that is, $\left(x^{*}, y^{*}\right) \in\left(T\left(\operatorname{gph} M_{r} ;(\bar{x}, \bar{y})\right)\right)^{-}=N\left(\operatorname{gph} M_{r} ;(\bar{x}, \bar{y})\right)$. Since $N\left(g p h M_{r} ;(\bar{x}, \bar{y})\right)$ is a closed convex cone in $E^{*} \times F^{*}$, we obtain that

$$
\mathrm{cl}_{w^{*}}\left(\mathbb{R}_{+} \partial \mathscr{T}_{M, \Omega}(\bar{x}, \bar{y})\right) \subset N\left(g p h M_{r} ;(\bar{x}, \bar{y})\right)
$$

Conversely, we have to prove that

$$
N\left(\operatorname{gph} M_{r} ;(\bar{x}, \bar{y})\right) \subset \mathrm{cl}_{w^{*}}\left(\mathbb{R}_{+} \partial \mathscr{T}_{M, \Omega}(\bar{x}, \bar{y})\right) .
$$

We will prove that the negative polar of $\mathrm{cl}_{w^{*}}\left(\mathbb{R}_{+} \partial \mathscr{T}_{M, \Omega}(\bar{x}\right.$, $\bar{y}))$ is included in $T\left(\operatorname{gph} M_{r} ;(\bar{x}, \bar{y})\right)=\left(N\left(\operatorname{gph} M_{r} ;(\bar{x}, \bar{y})\right)\right)^{-}$; that is, (by Theorem 12)

$$
\begin{aligned}
& \left(\mathrm{cl}_{w^{*}}\left(\mathbb{R}_{+} \partial \mathscr{T}_{M, \Omega}(\bar{x}, \bar{y})\right)\right)^{-} \\
& \quad \subset\left\{(h, k) \in E \times F: \mathscr{T}_{M, \Omega}^{\prime}((\bar{x}, \bar{y}) ;(h, k)) \leq 0\right\} .
\end{aligned}
$$

Note first that

$$
\left(\operatorname{cl}_{w^{*}}\left(\mathbb{R}_{+} \partial \mathscr{T}_{M, \Omega}(\bar{x}, \bar{y})\right)\right)^{-}=\left(\partial \mathscr{T}_{M, \Omega}(\bar{x}, \bar{y})\right)^{-} .
$$

So consider any $(h, k) \in\left(\partial \mathscr{T}_{M, \Omega}(\bar{x}, \bar{y})\right)^{-}$. Then, we have

$$
\left\langle\left(x^{*}, y^{*}\right),(h, k)\right\rangle \leq 0, \quad \forall\left(x^{*}, y^{*}\right) \in \partial \mathscr{T}_{M, \Omega}(\bar{x}, \bar{y}) .
$$


Hence, $\quad\left(\right.$ since $\left.\partial \mathscr{T}_{M, \Omega}(\bar{x}, \bar{y})\right) \neq \emptyset \quad$ and $\quad(\bar{x}, \bar{y}) \quad \in$ $\left.\operatorname{core}\left(\operatorname{dom} T_{M, \Omega}\right)\right)$

$$
\begin{aligned}
& \mathscr{T}_{M, \Omega}^{\prime}((\bar{x}, \bar{y}) ;(h, k)) \\
& =\sigma_{\partial \mathscr{T}_{M, \Omega}(\bar{x}, \bar{y})}(h, k) \\
& =\sup _{\left(x^{*}, y^{*}\right) \in \partial \mathscr{T}_{M, \Omega}(\bar{x}, \bar{y})}\left\langle\left(x^{*}, y^{*}\right),(h, k)\right\rangle \leq 0,
\end{aligned}
$$

which ensures by Theorem 12 that $(\bar{h}, \bar{k}) \in T\left(g p h M_{r} ;(\bar{x}, \bar{y})\right)$. Thus, completing the proof.

The following corollary extends Corollary 1 in [14] from the case of sets to the case of set-valued mappings and from normed vector spaces to Hausdorff Topological vector spaces. It says that if we put the calmness of $\mathscr{T}_{M, \Omega}$ instead of its l.s.c. and the assumption $0 \notin \partial \mathscr{T}_{M, \Omega}(\bar{x}, \bar{y})$ instead of the assumption $\partial \mathscr{T}_{M, \Omega}(\bar{x}, \bar{y}) \neq \emptyset$ in Theorem 14 we may remove the weak star closedness in the second equation in Theorem 14. Recall that $f$ is said to be calm at $\bar{u}$ if there exists a closed balanced neighborhood $V$ of zero and $U \in \mathcal{N}(\bar{u})$ such that

$$
|f(u)-f(\bar{u})| \leq \rho_{V}(\bar{u}-u), \quad \forall u \in U .
$$

Corollary 15. Let $E$ and $F$ Hausdorff topological vector spaces, $M: E \rightrightarrows F$ a set-valued mapping with a closed convex graph, $\Omega$ be a bounded convex set in $F$, and $(\bar{x}, \bar{y}) \notin g p h M$. If $\mathscr{T}_{M, \Omega}$ is calm at $(\bar{x}, \bar{y})$ with $0 \notin \partial \mathscr{T}_{M, \Omega}(\bar{x}, \bar{y})$, then one has

$$
N\left(\operatorname{gph} M_{r} ;(\bar{x}, \bar{y})\right)=\mathbb{R}_{+} \partial \mathscr{T}_{M, \Omega}(\bar{x}, \bar{y}) .
$$

Proof. Using the calmness of $\mathscr{T}_{M, \Omega}$ at $(\bar{x}, \bar{y})$ and BanachAlaoglu theorem (see Theorem 3.5 in [21]), we get the weak star compactness of $\partial^{C} \mathscr{T}_{M, \Omega}(\bar{x}, \bar{y})$ in $E^{*} \times F^{*}$. Therefore, the assumption $0 \notin \partial^{C} \mathscr{T}_{M, \Omega}(\bar{x}, \bar{y})$ with the weak star compactness of $\partial^{C} \mathscr{T}_{M, \Omega}(\bar{x}, \bar{y})$ ensures the weak star closedness of the cone generated by $\partial^{C} \mathscr{T}_{M, \Omega}(\bar{x}, \bar{y})$; that is, $\mathrm{cl}_{w^{*}}\left(\mathbb{R}_{+} \partial^{C} \mathscr{T}_{M, \Omega}(\bar{x}, \bar{y})\right)=\mathbb{R}_{+} \partial^{C} \mathscr{T}_{M, \Omega}(\bar{x}, \bar{y})$. Thus, the conclusion follows from Theorem 14 .

The next results depend on the nonemptiness of the minimum set for the minimal time function $\mathscr{T}_{M, \Omega}$ defined as follows

$$
\pi_{M(x), \Omega}(u):=\left\{y \in M(x): \mathscr{T}_{M, \Omega}(x, y)=\rho_{\Omega}(y-u)\right\} .
$$

We begin with the following lemma.

Lemma 16. Let $E$ and $F$ be two Hausdorff topological vector spaces, $M: E \rightrightarrows F$ a set-valued mapping with closed convex graph, $\Omega$ a bounded convex set in $F$, and $(\bar{x}, \bar{y}) \notin g p h M$ with $\mathscr{T}_{M, \Omega}(\bar{x}, \bar{y})<\infty$ and $\pi_{M(\bar{x}), \Omega}(\bar{y}) \neq \emptyset$. For every $y \in \pi_{M(\bar{x}), \Omega}(\bar{y})$ and for every $t \in(0,1]$, one has

$$
\mathscr{T}_{M, \Omega}(\bar{x}, t y+(1-t) \bar{y})=(1-t) \mathscr{T}_{M, \Omega}(\bar{x}, \bar{y}) .
$$
$(0,1]$.
Proof. Let $y \in \pi_{M(\bar{x}), \Omega}(\bar{y})$. Then $y \in M(\bar{x})$ with $\mathscr{T}_{M, \Omega}(\bar{x}, \bar{y})=$ $\rho_{\Omega}(y-\bar{y})<\infty$. First, we prove the inequality

$$
\begin{aligned}
& \mathscr{T}_{M, \Omega}(\bar{x}, t y+(1-t) \bar{y}) \\
& \quad \leq(1-t) \mathscr{T}_{M, \Omega}(\bar{x}, \bar{y}), \quad \forall t \in(0,1] .
\end{aligned}
$$

Put $\alpha:=\rho_{\Omega}(y-\bar{y})>0$, and fix any $t \in(0,1]$. Clearly, by definition of the Minkowski function, we can find for any $\epsilon>0$ some $\alpha_{0} \geq 0$ such that $y-\bar{y} \in \alpha_{0} \Omega$ and $\alpha_{0} \in[\alpha, \alpha+\epsilon)$, and so

$$
\begin{gathered}
y=t y+(1-t) \bar{y}+\alpha_{0}(1-t) \frac{(y-\bar{y})}{\alpha_{0}} \in M(\bar{x}) \\
\cap\left[t y+(1-t) \bar{y}+\alpha_{0}(1-t) \Omega\right] \neq \emptyset,
\end{gathered}
$$

which ensures that

$$
\begin{aligned}
\mathscr{T}_{M, \Omega} & (\bar{x}, t y+(1-t) \bar{y}) \\
& \leq \alpha_{0}(1-t)<(1-t)\left[\rho_{\Omega}(y-\bar{y})+\epsilon\right] \\
& \leq(1-t) \mathscr{T}_{M, \Omega}(\bar{x}, \bar{y})+\epsilon, \quad \forall \epsilon>0 .
\end{aligned}
$$

Taking $\epsilon \rightarrow 0^{+}$finishes the proof of the first inequality.

By the first part, we have $\mathscr{T}_{M, \Omega}(\bar{x}, t y+(1-t) \bar{y})<\infty$ whenever $\mathscr{T}_{M, \Omega}(\bar{x}, \bar{y})<\infty$ and so for any $\epsilon>0$, we can find some $w \in \Omega$, some $\delta>0$, and some $\delta_{0}>0$ such that

$$
\begin{gathered}
\mathscr{T}_{M, \Omega}(\bar{x}, t y+(1-t) \bar{y}) \\
\leq \delta<\mathscr{T}_{M, \Omega}(\bar{x}, t y+(1-t) \bar{y})+\epsilon, \\
t y+(1-t) \bar{y}+\delta w \in M(\bar{x}), \\
y-\bar{y} \epsilon \delta_{0} \Omega \quad \text { with } \quad 0<\rho_{\Omega}(y-\bar{y}) \leq \delta_{0}<\rho_{\Omega}(y-\bar{y})+\epsilon .
\end{gathered}
$$

Then, by convexity of $\Omega$, we have $\delta_{0} t\left((y-\bar{y}) / \delta_{0}\right)+\delta w \epsilon$ $\left(\delta_{0} t+\delta\right) \Omega$, and so

$$
\begin{gathered}
\bar{y}+t(y-\bar{y})+\delta w=\bar{y}+\delta_{0} t \frac{(y-\bar{y})}{\delta_{0}}+\delta w \in M(\bar{x}) \\
\cap\left[\bar{y}+\left(\delta_{0} t+\delta\right) \Omega\right] \neq \emptyset .
\end{gathered}
$$

Hence,

$$
\begin{aligned}
\mathscr{T}_{M, \Omega}(\bar{x}, \bar{y}) \\
\quad \leq \delta_{0} t+\delta \\
\quad<t\left(\rho_{\Omega}(y-\bar{y})+\epsilon\right)+\mathscr{T}_{M, \Omega}(\bar{x}, t y+(1-t) \bar{y})+\epsilon \\
\quad<t \mathscr{T}_{M, \Omega}(\bar{x}, \bar{y})+\mathscr{T}_{M, \Omega}(\bar{x}, t y+(1-t) \bar{y})+2 \epsilon,
\end{aligned}
$$

and so by taking $\epsilon \rightarrow 0^{+}$, we obtain that

$$
(1-t) \mathscr{T}_{M, \Omega}(\bar{x}, \bar{y}) \leq \mathscr{T}_{M, \Omega}(\bar{x}, t y+(1-t) \bar{y}) .
$$

This completes the proof of the lemma. 
We use this lemma to prove the following proposition on directional derivatives and convex subdifferentials of $\mathscr{T}_{M, \Omega}$ at points outside the graph.

Proposition 17. Assume that the assumptions of Lemma 16 are fulfilled. Then, for every $(\bar{x}, \bar{y}) \notin g p h M$ and for every $y \in \pi_{M(\bar{x}), \Omega}(\bar{y})$, one has

$$
\begin{gathered}
\mathscr{T}_{M, \Omega}^{\prime}((\bar{x}, \bar{y}) ;(0, y-\bar{y}))=-\mathscr{T}_{M, \Omega}(\bar{x}, \bar{y}), \\
\partial \mathscr{T}_{M, \Omega}(\bar{x}, \bar{y}) \subset\left\{\left(x^{*}, y^{*}\right) \in E^{*} \times F^{*}: \sigma_{\Omega}\left(-y^{*}\right)=1\right\} .
\end{gathered}
$$

Proof. By Lemma 16, we have

$$
\begin{aligned}
& T_{M, \Omega}((\bar{x}, \bar{y}) ;(0, y-\bar{y})) \\
& \quad=\lim _{t \rightarrow 0^{+}} t^{-1}\left[\mathscr{T}_{M, \Omega}(\bar{x}, \bar{y}+t(y-\bar{y}))-\mathscr{T}_{M, \Omega}(\bar{x}, \bar{y})\right] \\
& \quad=-\mathscr{T}_{M, \Omega}(\bar{x}, \bar{y}) .
\end{aligned}
$$

We turn now to show the inclusion

$$
\partial \mathscr{T}_{M, \Omega}(\bar{x}, \bar{y}) \subset\left\{\left(x^{*}, y^{*}\right) \in E^{*} \times F^{*}: \sigma_{\Omega}\left(-y^{*}\right)=1\right\} .
$$

Fix any $\left(x^{*}, y^{*}\right) \in \partial \mathscr{T}_{M, \Omega}(\bar{x}, \bar{y})$. Then

$$
\left\langle y^{*}, k\right\rangle \leq \mathscr{T}_{M, \Omega}^{\prime}((\bar{x}, \bar{y}) ;(0, k)), \quad \forall k \in F .
$$

By assumption, we have $\pi_{M(\bar{x}), \Omega}(\bar{y}) \neq \emptyset$. Then, there exists $y_{0} \in \pi_{M(\bar{x}), \Omega}(\bar{y})$; that is, $y_{0} \in M(\bar{x})$ with $\mathscr{T}_{M, \Omega}(\bar{x}, \bar{y})=$ $\rho_{\Omega}\left(y_{0}-\bar{y}\right) \neq 0$. By the first part, we obtain that

$$
\begin{aligned}
\left\langle y^{*}, y_{0}-\bar{y}\right\rangle & \leq \mathscr{T}_{M, \Omega}^{\prime}\left((\bar{x}, \bar{y}) ;\left(0, y_{0}-\bar{y}\right)\right) \\
& =-\mathscr{T}_{M, \Omega}(\bar{x}, \bar{y})=-\rho_{\Omega}\left(y_{0}-\bar{y}\right),
\end{aligned}
$$

and so

$$
\left\langle-y^{*}, \frac{y_{0}-\bar{y}}{\rho_{\Omega}\left(y_{0}-\bar{y}\right)}\right\rangle \geq 1,
$$

since $\rho_{\Omega}\left(y_{0}-\bar{y}\right) \neq 0$, because $\bar{y} \notin M(\bar{x})$. Thus, for any $\epsilon>0$, there exist $w \in \Omega$ and $\alpha_{0}>0$ such that

$$
0<\rho_{\Omega}\left(y_{0}-\bar{y}\right) \leq \alpha_{0}<\rho_{\Omega}\left(y_{0}-\bar{y}\right)+\epsilon
$$

and $y_{0}-\bar{y} \in \alpha_{0} \Omega$. Thus,

$$
\begin{gathered}
\sigma_{\Omega}\left(-y^{*}\right) \geq\left\langle-y^{*}, \frac{y_{0}-\bar{y}}{\alpha_{0}}\right\rangle \\
=\frac{\rho_{\Omega}\left(y_{0}-\bar{y}\right)}{\alpha_{0}}\left\langle-y^{*}, \frac{y_{0}-\bar{y}}{\rho_{\Omega}\left(y_{0}-\bar{y}\right)}\right\rangle \\
\geq \frac{\rho_{\Omega}\left(y_{0}-\bar{y}\right)}{\alpha_{0}} \geq \frac{\rho_{\Omega}\left(y_{0}-\bar{y}\right)}{\rho_{\Omega}\left(y_{0}-\bar{y}\right)+\epsilon},
\end{gathered}
$$

and hence,

$$
\sigma_{\Omega}\left(-y^{*}\right) \geq 1-\frac{\epsilon}{\rho_{\Omega}\left(y_{0}-\bar{y}\right)+\epsilon}, \quad \forall \epsilon>0 .
$$

Taking $\epsilon \rightarrow 0^{+}$yields

$$
\sigma_{\Omega}\left(-y^{*}\right) \geq 1 .
$$

Let us prove the reverse inequality

$$
\sigma_{\Omega}\left(-y^{*}\right) \leq 1 .
$$

Let $\left(t_{j}\right)_{j}$ be a net in $(0, \infty)$ converging to 0 and satisfying

$$
\begin{aligned}
& \mathscr{T}_{M, \Omega}^{\prime}((\bar{x}, \bar{y}) ;(0, k)) \\
& \quad=\lim _{j} t_{j}^{-1}\left[\mathscr{T}_{M, \Omega}\left(\bar{x}, \bar{y}+t_{j} k\right)-\mathscr{T}_{M, \Omega}(\bar{x}, \bar{y})\right] .
\end{aligned}
$$

By Lemma 10, we have, for $r:=\mathscr{T}_{M, \Omega}(\bar{x}, \bar{y})$, the following inequality

$$
\begin{aligned}
& \mathscr{T}_{M, \Omega}(x, y)-\mathscr{T}_{M, \Omega}(\bar{x}, \bar{y}) \\
& \quad \leq \mathscr{T}_{M_{r}, \Omega}(x, y)-\mathscr{T}_{M_{r}, \Omega}(\bar{x}, \bar{y}), \quad \forall(x, y) \in E \times F,
\end{aligned}
$$

which ensures that

$$
\begin{aligned}
t_{j}^{-1} & {\left[\mathscr{T}_{M, \Omega}\left(\bar{x}, \bar{y}+t_{j} k\right)-\mathscr{T}_{M, \Omega}(\bar{x}, \bar{y})\right] } \\
& \leq t_{j}^{-1} \mathscr{T}_{M_{r}, \Omega}\left(\bar{x}, \bar{y}+t_{j} k\right) \\
& =t_{j}^{-1} \inf _{u \in M_{r}(\bar{x})} \rho_{\Omega}\left(u-\left(\bar{y}+t_{j} k\right)\right) \\
& \leq t_{j}^{-1} \rho_{\Omega}\left(\bar{y}-\left(\bar{y}+t_{j} k\right)\right)=\rho_{\Omega}(-k),
\end{aligned}
$$

and so

$$
t_{j}^{-1}\left[\mathscr{T}_{M, \Omega}\left(\bar{x}, \bar{y}+t_{j} k\right)-\mathscr{T}_{M, \Omega}(\bar{x}, \bar{y})\right] \leq \rho_{\Omega}(-k) .
$$

Taking the limit on $j$, we get that

$$
\begin{aligned}
& \mathscr{T}_{M, \Omega}^{\prime}((\bar{x}, \bar{y}) ;(0, k)) \\
& \quad=\lim _{j} t_{j}^{-1}\left[\mathscr{T}_{M, \Omega}\left(\bar{x}, \bar{y}+t_{j} k\right)-\mathscr{T}_{M, \Omega}(\bar{x}, \bar{y})\right] \leq \rho_{\Omega}(-k) .
\end{aligned}
$$

Using the fact that $\left(x^{*}, y^{*}\right) \in \partial \mathscr{T}_{M, \Omega}(\bar{x}, \bar{y})$, we write

$$
\begin{aligned}
\left\langle-y^{*},-k\right\rangle & =\left\langle\left(x^{*}, y^{*}\right) ;(0, k)\right\rangle \leq \mathscr{T}_{M, \Omega}((\bar{x}, \bar{y}) ;(0, k)) \\
& \leq \rho_{\Omega}(-k), \quad \forall k \in F .
\end{aligned}
$$

Fix any $v \in \Omega$, and take $k:=-v$ in the previous inequality we get

$$
\left\langle-y^{*}, v\right\rangle=\left\langle-y^{*},-k\right\rangle \leq \rho_{\Omega}(v) \leq 1 .
$$

This ensures that $\sigma_{\Omega}\left(-y^{*}\right) \leq 1$, and hence, the proof is finished.

The following theorem establishes another relationship between the convex subdifferential of $\mathscr{T}_{M, \Omega}$ and the convex normal cone of $M_{r}$ at points outside the graph $g$ ph $M$. 
Theorem 18. Let $E$ and $F$ be two Hausdorff topological vector spaces, $M$ a set-valued mapping with nonempty closed values of $F, \Omega$ be a bounded convex set in $F$, and $(\bar{x}, \bar{y}) \notin g p h M$. Assume that $\pi_{M(\bar{x}), \Omega}(\bar{y}) \neq \emptyset$. Then, one has

$$
\begin{aligned}
\partial \mathscr{T}_{M, \Omega}(\bar{x}, \bar{y}) & \subset N\left(\operatorname{gph} M_{r} ;(\bar{x}, \bar{y})\right) \\
& \cap\left\{\left(x^{*}, y^{*}\right) \in E^{*} \times F^{*}: \sigma_{\Omega}\left(-y^{*}\right)=1\right\} .
\end{aligned}
$$

If, in addition, $\mathscr{T}_{M, \Omega}$ is calm at $(\bar{x}, \bar{y})$, then one has

$$
\begin{aligned}
\partial \mathscr{T}_{M, \Omega}(\bar{x}, \bar{y})=N\left(\operatorname{gph} M_{r} ;(\bar{x}, \bar{y})\right) \\
\quad \cap\left\{\left(x^{*}, y^{*}\right) \in E^{*} \times F^{*}: \sigma_{\Omega}\left(-y^{*}\right)=1\right\} .
\end{aligned}
$$

Proof. The first inclusion follows directly from the proof of Theorem 14 and Proposition 17. Let us prove the reverse inclusion. Fix any $\left(x^{*}, y^{*}\right) \in N\left(g p h M_{r} ;(\bar{x}, \bar{y})\right)$ with $\sigma_{\Omega}\left(-y^{*}\right)=1$. First, let us check that $0 \notin \partial \mathscr{T}_{M, \Omega}(\bar{x}, \bar{y})$. Indeed, by the assumption $\pi_{M(\bar{x}), \Omega}(\bar{y}) \neq \emptyset$ and Proposition 17, there exists $z_{0} \in \pi_{M(\bar{x}), \Omega}(\bar{y})$ such that

$$
\mathscr{T}_{M, \Omega}^{\prime}\left((\bar{x}, \bar{y}) ;\left(0, z_{0}-\bar{y}\right)\right)=-\mathscr{T}_{M, \Omega}(\bar{x}, \bar{y})<0,
$$

which ensures that $0 \notin \partial \mathscr{T}_{M, \Omega}(\bar{x}, \bar{y})$. Therefore, by Corollary 15, there exists some $\lambda>0$ and $\left(u^{*}, v^{*}\right) \in$ $\partial \mathscr{T}_{M, \Omega}(\bar{x}, \bar{y})$, such that $\left(x^{*}, y^{*}\right)=\lambda\left(u^{*}, v^{*}\right)$. Using the first part of the proof, we obtain that $\sigma_{\Omega}\left(-v^{*}\right)=1$, and so by combining this equality with the assumption $\sigma_{\Omega}\left(-y^{*}\right)=1$, we get $\lambda=1$. Thus completing the proof.

\section{Application of $\mathscr{T}_{M, \Omega}$ : Calmness and Exact Penalization}

The primary goal in the present section is to make clear that the scalar function $\mathscr{T}_{M, \Omega}$ can also be a powerful tool in the study of the calmness property of optimization problems in Hausdorff topological vector spaces. Here, we are interested in the concept of calmness of perturbed optimization problems with a constraint defined by a set used and studied by Burke $[22,23]$ in normed vector spaces. We will adapt his definition for a general perturbed problem with a constraint defined by a set-valued mapping in Hausdorff topological vector spaces, and we will prove that it is equivalent to the existence of an exact penalization in terms of the minimal time function associated with the set-valued mapping defining the constraint of the problem.

Consider the problem $(\mathscr{P})$, which consists in minimizing the function $f$ over all $x \in E$ satisfying $0 \in M(x)$,

$$
(\mathscr{P}) \begin{cases}\text { minimize } & f(x) \\ \text { subject to } & 0 \in M(x),\end{cases}
$$

where $M: E \rightrightarrows F$ is a closed set-valued mapping between two normed vector spaces $E$ and $F$ and $f: E \rightarrow \mathbb{R} \cup$ $\{+\infty\}$ is an extended real-valued function. We begin with the definition of calmness.
Definition 19. Let $f, M, E$, and $F$ be as in the statement of $(\mathscr{P})$, and consider the following perturbed problem

$$
\left(\mathscr{P}_{y}\right) \begin{cases}\text { minimize } & f(x) \\ \text { subject to } & y \in M(x) .\end{cases}
$$

Let $S: F \rightrightarrows E$ be the feasible set-valued mapping associated with $\left(\mathscr{P}_{y}\right)$; that is,

$$
S(y):=\{x \in \operatorname{dom} f: y \in M(x)\} .
$$

Let $(\bar{x}, \bar{y}) \in g p h S$ and let $\Omega$ be a bounded set in $F$. One will say that the problem $\left(\mathscr{P}_{\bar{y}}\right)$ is calm at $\bar{x}$ with respect to $\Omega$ if there exist a constant $\bar{\alpha} \geq 0$ and $X \in \mathscr{N}_{E}(\bar{x})$ such that for every $x \in X$ and any $y \in M(x)$ one has

$$
f(\bar{x}) \leq f(x)+\bar{\alpha} \rho_{\Omega}(y-\bar{y}) .
$$

The constant $\bar{\alpha}$ and $X$ are called the modulus of calmness and neighborhood of calmness for $\left(\mathscr{P}_{\bar{y}}\right)$ at $\bar{x}$, respectively.

Remark 20. When $F$ is assumed to be normed, the above definition coincides with the definition used in $[22,23]$ by taking $\Omega$ to be the closed unit ball in $F$. Observe that if $\left(\mathscr{P}_{\bar{y}}\right)$ is calm at $\bar{x}$ with respect to a bounded set $\Omega$, then $\bar{x}$ is necessarily a local solution to $\left(\mathscr{P}_{\bar{y}}\right)$.

For any problem $\left(\mathscr{P}_{y}\right)$, any real number $\alpha \geq 0$, and any bounded set $\Omega$, we will associate the function $P_{\alpha, y, \Omega}$ defined by

$$
P_{\alpha, y, \Omega}(x):=f(x)+\alpha \mathscr{T}_{M, \Omega}(x, y) .
$$

In the following theorem we state our main result in this section. It establishes a relationship between the calmness property and the existence of an exact penalization of the general perturbed problem $\left(\mathscr{P}_{\bar{y}}\right)$ in terms of the minimal time function to images associated with the set-valued mapping $M$ defining the constraint of the problem.

Theorem 21. Let $(\bar{x}, \bar{y}) \in$ gphS. If $\left(\mathscr{P}_{\bar{y}}\right)$ is calm at $\bar{x}$ with respect to $\Omega$ with modulus $\bar{\alpha}$ and neighborhood $X \in \mathcal{N}_{E}(\bar{x})$, then $\bar{x}$ is a minimum over $X$ of the function $P_{\alpha, \bar{y}, \Omega}$, for all $\alpha \geq \bar{\alpha}$. If, in addition, $\Omega$ is convex and $0 \in \Omega$, the converse holds.

Proof. Let $\epsilon>0$. Given any $x \in X \cap \operatorname{dom} M \neq \emptyset$. Then there exist $t_{0} \geq 0$ and $w \in \Omega$ such that $\bar{y}+t_{0} w \in M(x)$ and $\mathscr{T}_{M, \Omega}(\bar{x}, \bar{y}) \leq t_{0}<\mathscr{T}_{M, \Omega}(\bar{x}, \bar{y})+\epsilon$. Thus, if $\alpha \geq \bar{\alpha}$, we obtain from the calmness hypothesis that

$$
f(\bar{x}) \leq f(x)+\alpha \rho_{\Omega}(y-\bar{y}), \quad \forall x \in X, \forall y \in M(x) .
$$

Fix any $x \in X$. Then by taking $y:=\bar{y}+t_{0} w$ in the previous inequality and observing that $\rho_{\Omega}(w) \leq 1$ we obtain that

$$
\begin{aligned}
f(\bar{x}) & \leq f(x)+\alpha \rho_{\Omega}\left(t_{0} w\right) \leq f(x)+t_{0} \alpha \rho_{\Omega}(w) \\
& \leq f(x)+t_{0} \alpha \leq f(x)+\alpha \mathcal{T}_{M, \Omega}(\bar{x}, \bar{y})+\alpha \epsilon .
\end{aligned}
$$

Hence

$$
P_{\alpha, \bar{y}, \Omega}(\bar{x})=f(\bar{x}) \leq P_{\alpha, \bar{y}, \Omega}(x)+\alpha \epsilon, \quad \forall x \in X .
$$


Since $\epsilon>0$ was taken arbitrarily, the direct implication is established by taking $\epsilon \rightarrow 0$.

Conversely, we assume that $\Omega$ is convex and $0 \in \Omega$. Fix any $x \in X$ and any $y \in M(x)$. We claim first the inequality $\mathscr{T}_{M, \Omega}(x, \bar{y}) \leq \rho_{\Omega}(y-\bar{y})$. Clearly the case $\rho_{\Omega}(y-\bar{y})=\infty$ is obvious. So we assume that $\rho_{\Omega}(y-\bar{y})<\infty$. Since $y \in M(x)$, we have $\mathscr{T}_{M, \Omega}(x, y)=0$. Then by the finiteness of $\mathscr{T}_{M, \Omega}(x, y)$ we can find for any $\epsilon>0$ some $\lambda_{0} \geq 0$ such that

$$
\begin{aligned}
0 & =\mathscr{T}_{M, \Omega}(x, y) \\
& \leq \lambda_{0}<\mathscr{T}_{M, \Omega}(x, y)+\epsilon=\epsilon, \quad\left(y+\lambda_{0} \Omega\right) \cap M(x) \neq \emptyset .
\end{aligned}
$$

Hence, there exists $w \in \Omega$ such that $y+\lambda_{0} w \in M(x)$. Therefore, for any $v \in \Omega$ and any $\beta>0$, we have

$$
\begin{aligned}
y & +\lambda_{0} w \\
& =y-\beta v+\left(\lambda_{0} w+\beta v\right) \\
& =y-\beta v+\left(\lambda_{0}+\beta\right)\left(\frac{\lambda_{0}}{\lambda_{0}+\beta} w+\frac{\beta}{\lambda_{0}+\beta} v\right) \in M(x) .
\end{aligned}
$$

Since $\Omega$ is convex, we have $\left(\lambda_{0} /\left(\lambda_{0}+\beta\right)\right) w+(\beta /(\lambda+\beta)) v \in$ $\Omega$, and so $\left(y-\beta v+\left(\lambda_{0}+\beta\right) \Omega\right) \cap M(x) \neq \emptyset$, which ensures by the definition of the minimal time function that

$$
\mathscr{T}_{M, \Omega}(x, y-\beta v) \leq \lambda_{0}+\beta<\epsilon+\beta, \quad \forall \epsilon>0 .
$$

Since $\rho_{\Omega}(y-\bar{y})<\infty$, we let $\beta:=\rho_{\Omega}(y-\bar{y})+\epsilon<\infty$ and $v:=(y-\bar{y}) / \beta$. Since $\Omega$ is convex and $0 \in \Omega$, we have $\left\{h \in E: \rho_{\Omega}(h)<1\right\} \subset \Omega$, and hence $v \in \Omega$. Thus, (127) ensures that

$$
\begin{aligned}
\mathscr{T}_{M, \Omega}(x, \bar{y}) & =\mathscr{T}_{M, \Omega}(x, y-\beta v)<\epsilon+\beta \\
& =\rho_{\Omega}(y-\bar{y})+2 \epsilon, \quad \forall \epsilon>0 .
\end{aligned}
$$

After taking $\epsilon \rightarrow 0$, this inequality ensures the desired inequality; that is, $\mathscr{T}_{M, \Omega}(x, \bar{y}) \leq \rho_{\Omega}(y-\bar{y})$. Therefore, for any $x \in X$ and any $y \in M(x)$, we have

$$
\begin{aligned}
f(\bar{x}) & =P_{\bar{\alpha}, \bar{y}, \Omega}(\bar{x}) \leq P_{\bar{\alpha}, \bar{y}, \Omega}(x)=f(x)+\bar{\alpha} \mathscr{T}_{M, \Omega}(x, \bar{y}) \\
& \leq f(x)+\bar{\alpha} \rho_{\Omega}(y-\bar{y}) .
\end{aligned}
$$

Hence, $\left(\mathscr{P}_{\bar{y}}\right)$ is calm at $\bar{x}$ with respect to $\Omega$, and the proof of the theorem is complete.

\section{Acknowledgment}

The author extends his appreciations to the Deanship of Scientific Research at King Saud University for funding the work through the research group project no. RGP-VPP-024.

\section{References}

[1] M. Bounkhel, "On subdifferentials of a minimal time function in Hausdorff topological vector spaces," Applicable Analysis: An International Journal, 2013.
[2] M. Bounkhel, "On subdifferentials of a minimal time function at points outside the target in Hausdorff topological vector spaces," Journal of Convex Analysis, 2013.

[3] M. Bounkhel, "Directional Lipschitzness of minimal time functions in Hausdorff topological vector spaces," Set-Valued and Variational Analysis, 2013.

[4] P. Cannarsa and C. Sinestrari, Semiconcave Functions, Hamilton-Jacobi Equations, and Optimal Control, Birkhäuser, Boston, Mass, USA, 2004.

[5] G. Colombo and P. R. Wolenski, "The subgradient formula for the minimal time function in the case of constant dynamics in Hilbert space," Journal of Global Optimization, vol. 28, no. 3-4, pp. 269-282, 2004.

[6] G. Colombo and P. R. Wolenski, "Variational analysis for a class of minimal time functions in Hilbert spaces," Journal of Convex Analysis, vol. 11, no. 2, pp. 335-361, 2004.

[7] F. S. De Blasi and J. Myjak, "On a generalized best approximation problem," Journal of Approximation Theory, vol. 94, no. 1, pp. 54-72, 1998.

[8] Y. He and K. F. Ng, "Subdifferentials of a minimum time function in Banach spaces," Journal of Mathematical Analysis and Applications, vol. 321, no. 2, pp. 896-910, 2006.

[9] Y. Jiang and Y. He, "Subdifferentials of a minimal time function in normed spaces," Journal of Mathematical Analysis and Applications, vol. 358, no. 2, pp. 410-418, 2009.

[10] B. S. Mordukhovich and N. M. Nam, "Limiting subgradients of minimal time functions in Banach spaces," Journal of Global Optimization, vol. 46, no. 4, pp. 615-633, 2010.

[11] N. M. Nam, M. C. Villalobos, and N. T. An, "Minimal time functions and the smallest intersecting ball problem with unbounded dynamics," Journal of Optimization Theory and Applications, vol. 154, no. 3, pp. 768-791, 2012.

[12] P. Soravia, "Generalized motion of a front propagating along its normal direction: a differential games approach," Nonlinear Analysis. Theory, Methods \& Applications, vol. 22, no. 10, pp. 1247-1262, 1994.

[13] P. R. Wolenski and Y. Zhuang, "Proximal analysis and the minimal time function," SIAM Journal on Control and Optimization, vol. 36, no. 3, pp. 1048-1072, 1998.

[14] F. H. Clarke, Optimization and Nonsmooth Analysis, John Wiley \& Sons, New York, NY, USA, 1983.

[15] M. Bounkhel, Regularity Concepts in Nonsmooth Analysis. Theory and Applications, vol. 59 of Springer Optimization and Its Applications, Springer, New York, NY, USA, 2012.

[16] Y. Jiang and Y. He, "Subdifferential properties for a class of minimal time functions with moving target sets in normed spaces," Applicable Analysis, vol. 91, no. 3, pp. 491-502, 2012.

[17] W. Schirotzek, Nonsmooth Analysis, Universitext, Springer, Berlin, Germany, 2007.

[18] J. V. Burke, M. C. Ferris, and M. Qian, "On the Clarke subdifferential of the distance function of a closed set," Journal of Mathematical Analysis and Applications, vol. 166, no. 1, pp. 199-213, 1992.

[19] M. Bounkhel and L. Thibault, "On various notions of regularity of sets in nonsmooth analysis," Nonlinear Analysis. Theory, Methods \& Applications, vol. 48, no. 2, pp. 223-246, 2002.

[20] M. Bounkhel, "Scalarization of the normal Fréchet regularity of set-valued mappings," New Zealand Journal of Mathematics, vol. 33, no. 2, pp. 129-146, 2004.

[21] W. Rudin, Functional Analysis, McGraw-Hill, New York, NY, USA, 1973. 
[22] J. V. Burke, "An exact penalization viewpoint of constrained optimization," SIAM Journal on Control and Optimization, vol. 29, no. 4, pp. 968-998, 1991.

[23] J. V. Burke, "Calmness and exact penalization," SIAM Journal on Control and Optimization, vol. 29, no. 2, pp. 493-497, 1991. 


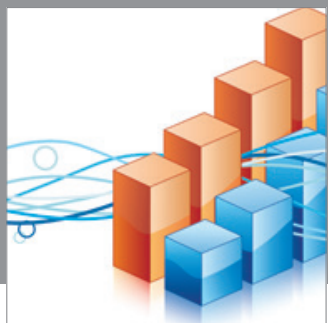

Advances in

Operations Research

mansans

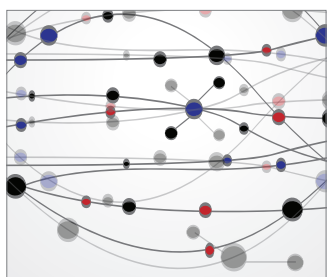

The Scientific World Journal
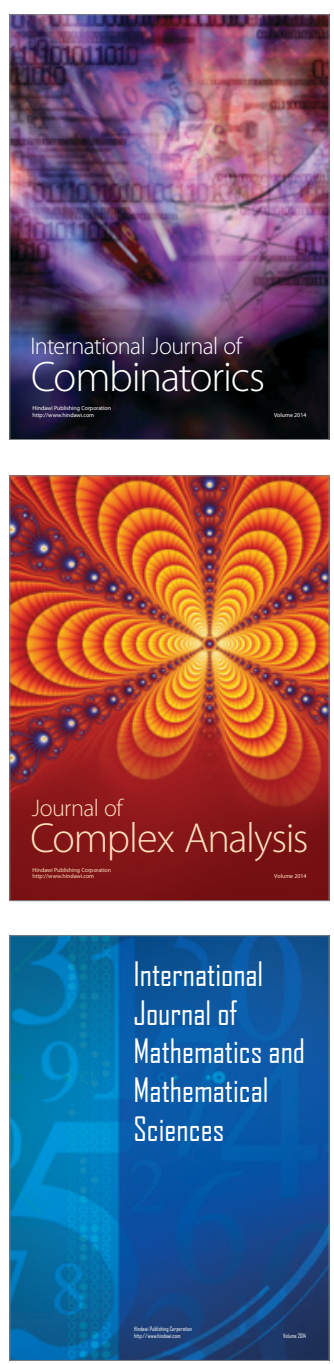
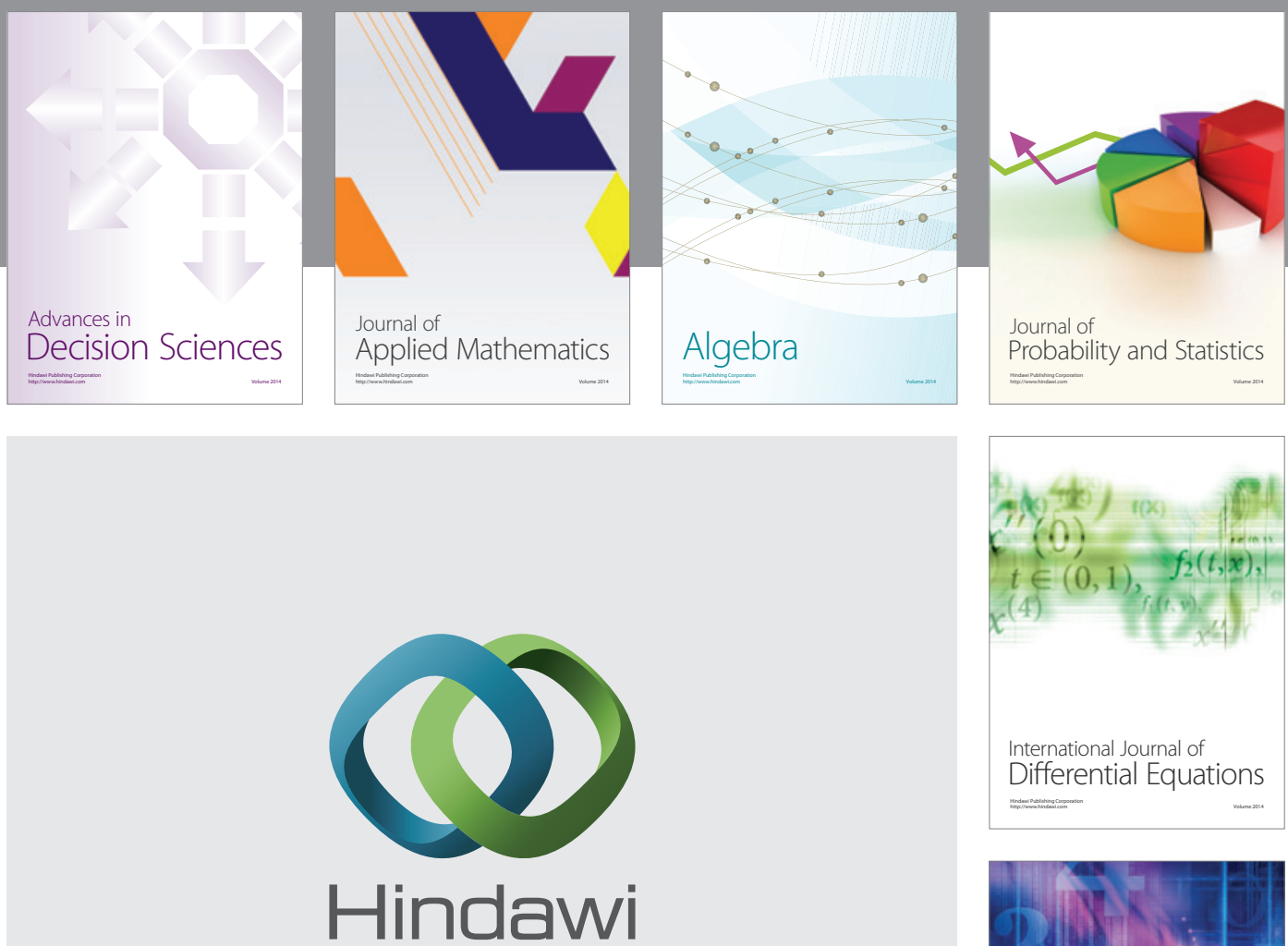

Submit your manuscripts at http://www.hindawi.com
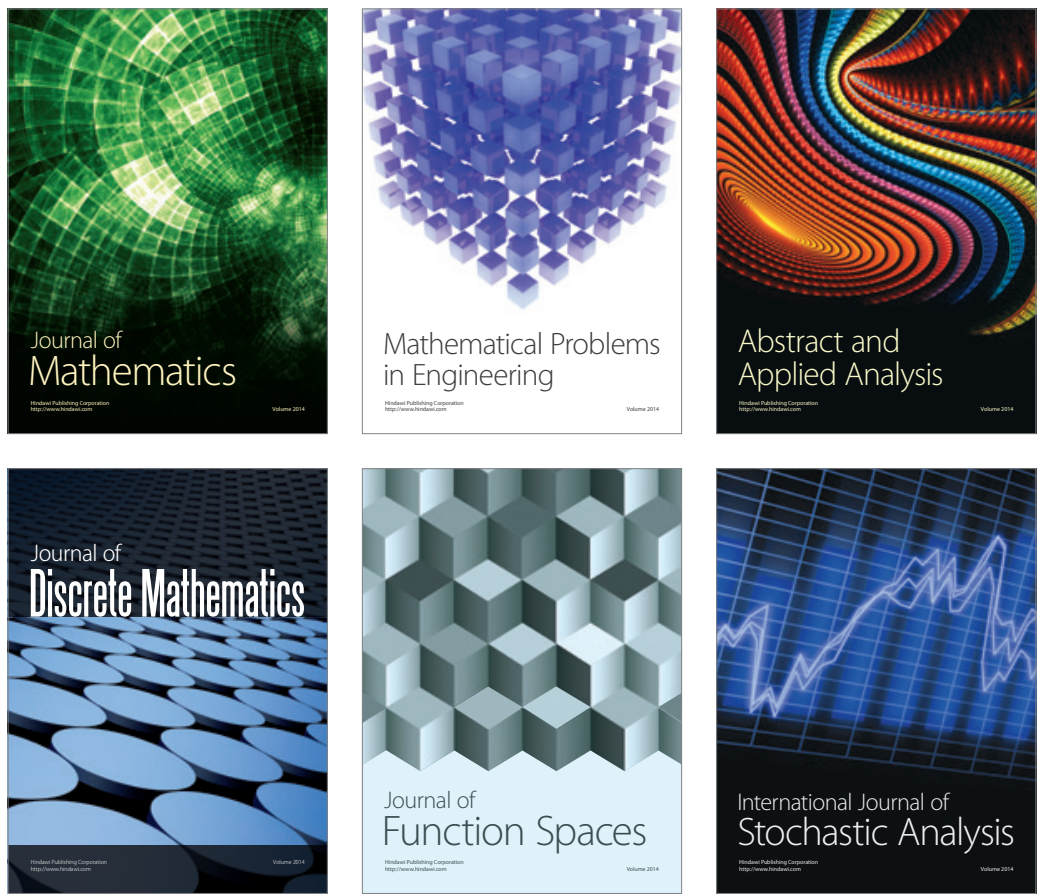

Journal of

Function Spaces

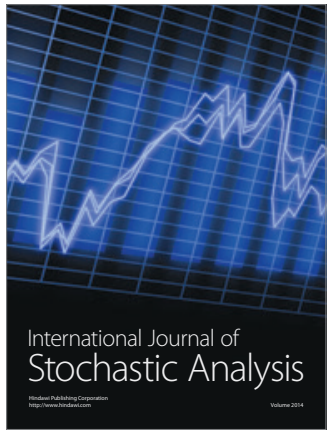

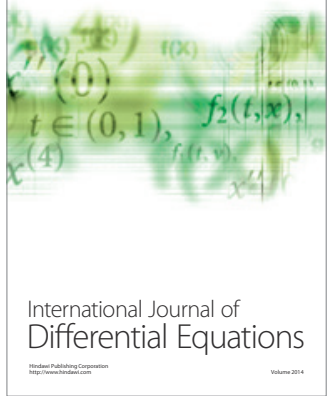
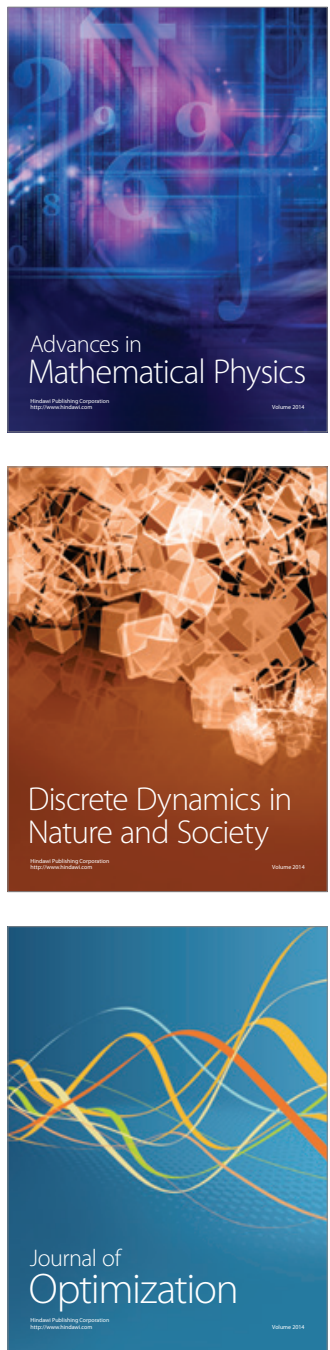